\title{
THE IMB PHOTOMULTIPLIER TEST FACILITY *
}

\author{
C.R. WUEST **, R.M. BIONTA, G. BLEWITT, C.B. BRATTON, B.G. CORTEZ, S. ERREDE, \\ G.W. FOSTER, W. GAJEWSKI, M. GOLDHABER, J. GREENBERG, T.W. JONES, W.R. KROPP, \\ J.G. LEARNED, E. LEHMANN, J.M. LoSECCO, P.V. RAMANA MURTHY, H.S. PARK, F. REINES, \\ J. SCHULTZ, E. SHUMARD, D. SINCLAIR, D.W. SMITH, H.W. SOBEL, J.L. STONE, L.R. SULAK, \\ R. SVOBODA and J.C. VAN DER VELDE
}

The University of California at Irvine, Irvine, California 92717, USA;

The University of Michigan, Ann Arbor, Michigan 48109, USA;

Brookhaven National Laboratory, Upton, New York 11973, USA;

California Institute of Technology, Pasadena, California 91125, USA;

Cleveland State University, Cleveland, Ohio 44115, USA;

The University of Hawaii, Honolulu, Hawaii 96822, USA;

University College, London WC1E 6BT, UK

Received 18 February 1985

An automatic system for testing up to 32 photomultiplier tubes (PMs) simultaneously under single photon counting conditions has been used to measure characteristics of more than 2500 PMs for use in the Irvine-Michigan-Brookhaven (IMB) proton decay experiment, 2048 tubes (64 EMI 9834B 8" diameter, and 1984 EMI 9870B 5" diameter) were selected for use in the $8000 \mathrm{~m}^{3} \mathrm{IMB}$ water Cherenkov detector, now in operation for over a year. The PM test system is described and results of testing are presented along with PM performance in the IMB detector over the last year. In general, we find that the tube characteristics have smaller fluctuations than expected and that the tubes have proven to be reliable under rugged handling and operating conditions. On the basis of our experience, we make suggestions as to new industry standards for PMs to be used in particle counting.

\section{Introduction}

The Irvine-Michigan-Brookhaven (IMB) water Cherenkov detector for nucleon decay studies and related physics [1] has been in successful operation for over one year and is providing new insights into the nature of the fundamental structure of matter. Specifically, the detector is designed to search for evidence of the decay of protons and neutrons (the source of which is the water itself) due to the hypothesized transformation of a quark-quark pair into an antilepton-antiquark pair within the nucleon's 3 quark structure. This class of transformations arises naturally from the theoretical extension of the "standard model" unification of electromagnetic and weak forces [2] (verified recently by the discovery of the $W$ and $Z$ bosons at CERN [3]) to the so-called Grand Unified Theories (GUTs), which

* Work performed under the auspices of the United States Department of Energy at Lawrence Livermore National Laboratory under Contract No. W-7405-Eng-48.

** Now at Lawrence Livermore National Laboratory, Livermore, CA 94550, USA. incorporate the strong interactions associated with quarks and gluons.

Evidence of nucleon decay is sought in the Cherenkov light cones generated by any relativistic charged decay products. Simple kinematics constrains the possible geometries and durations of these cones and allows a search for specific decay modes as confirmation of a particular theory. For example, the minimal SU(5) GUTs [4] predicts proton decay into positron plus neutral pion to be dominant. The decay will appear as two nearly equal energy back-to-back rings of light when the partially filled cones are projected onto planes perpendicular to each track's direction, equidistant from the proton decay point of origin or yertex.

Results for specific decay modes in the IMB detector have been presented elsewhere and will not be discussed here [5]. Instead, we will present an overview of the photomultiplier tubes (PMs) used in the IMB detector to image the faint traces of light associated with any charged particles traversing the detector at speeds greater than $0.75 c$, the speed of light in water. This paper is one in a series of overviews detailing various experimental systems in the IMB detector. 


\section{History}

In 1979, at the time the IMB experiment was proposed, it was generally recognized that a water Cherenkov detector of sufficient mass would be well suited to the detection of the new class of postulated nucleon decays associated with the new grand unified theories. It was determined that a detector of about $10000 \mathrm{t}$ mass instrumented with 2400 photomultiplier tubes on a one meter lattice surface array would provide the necessary resolution in position and energy to unambiguously sense most proton decay events. The detector performance would be significantly enhanced if the PMs were as large as possible, to increase energy resolution and sensitivity, and exhibited sufficiently small timing jitter to increase position resolution. The only PM which promised both was the EMI 9870B 5" diameter hemispherical PM and the EMI $98348^{\prime \prime}$ diameter hemispherical PM [6]. Other PM manufacturers were invited to enter the competition for supplying PMs to the IMB experiment, but only Hamamatsu TV Company was willing to undertake the necessary development work. Their $5^{\prime \prime}$ hemispherical tube was released too late to be used in the detector.

\section{The photomultiplier tube}

Fig. 1 shows the 5" PM. The 8" PM is similar to the $5^{\prime \prime}$, having a scaled up glass envelope with the same dynode structure. The tubes are very similar in design, but differ somewhat in operating characteristics. Both types of PMs are high gain (a factor of 10-100 million amplification, typically) with a "venetian blind" dynode structure and a bialkali photocathode. The glass envelope is about $2 \mathrm{~mm}$ thick at the face of the tube and is made of borosilicate (pyrex).

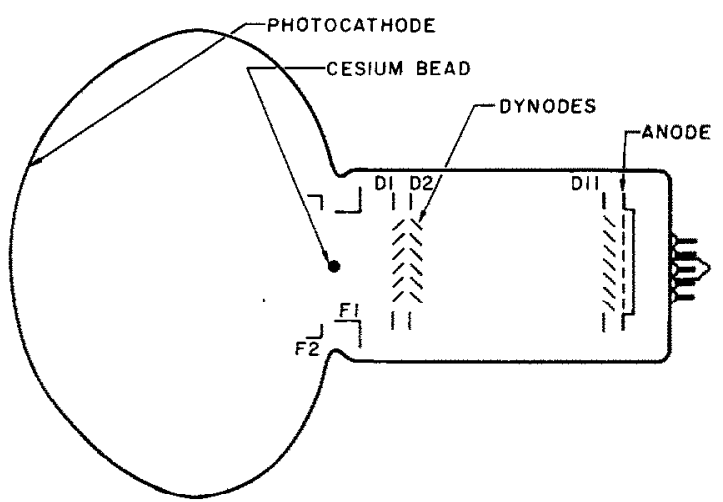

Fig. 1. The EMI $9870 \mathrm{~B}$ photomultiplier tube. The major features are the large area hemispherical window, independent focusing rings, $F 1$ and $F 2$, and the 11-stage venetian blind dynode chain.
The photocathode $(\mathrm{pc})$ is a semi-transparent layer of material deposited on the inside front hemisphere of the glass envelope. The material is a mixture of antimony, cesium, and rubidium (bialkali), which has a high sensitivity to photons in the wavelength region $300-500 \mathrm{~nm}$. The typical spectral response of bialkali is shown in fig. 2 as measured by the manufacturer. Photons striking the pc material have high probability of interacting and knocking electrons out via the photoelectric effect. The electrons are typically emitted with about $1 \mathrm{eV}$ of kinetic energy (compared to $2.5-4 \mathrm{eV}$ incident photon energy) and are distributed isotropically in emission angle with respect to the pc surface [7]. The PMs in the IMB detector are run with the pc at ground potential (positive polarity) to insure safe and reliable operation when immersed in water. The $\mathrm{pc}$ is not $100 \%$ efficient, i.e., not all incident photons interact with the pe to give an electron (an electron emitted from the pc surface is called a photoelectron or pe). Some photons pass through the pc and can be reflected back to the pc by means of an aluminium coating deposited on the inside back hemisphere of the PM.

The response of the $p c$ to incident photons is called the quantum efficiency (qe), the probability that an incident photon will knock out a pe from the pc. In practice, qe is not measured using a source of single photons. Instead, a source of light with a known emissivity and spectral character is used and an output signal (voltage, current or charge) is measured. In the PM industry manufacturers typically measure the total current resulting from a given continuous light source and using the entire dynode structure as a collector. This diode response gives a very optimistic value for the tube quantum efficiency, and manufacturers often report nothing about the collection efficiency, which is typically about $50 \%$. If the light level is low enough so that it is improbable for more than one photon to emit a photoelectron then statistical methods can be used to calibrate the tube response. By filtering the light through

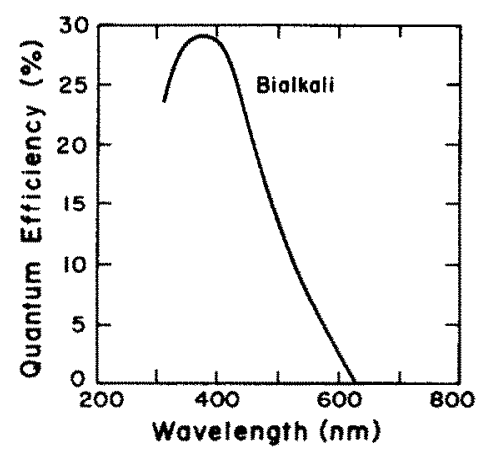

Fig. 2. Manufacturer's curve of typical bialkali photocathode quantum efficiency. Cutoff at $300 \mathrm{~nm}$ is due to pyrex glass window. 
narrow bandpass filters a spectral response curve as in fig. 2 is obtained. A system was developed at the University of California, Irvine using a calibrated photodiode as a reference to measure PM qe. This measurement will be described in greater detail in this paper.

The overall efficiency of a PM is also affected by collection and dynode efficiencies. Collection efficiency (ce) is the major effect, because once an electron is input to the dynode, it has a high probability (greater than $99 \%$ ) of getting amplified. Collection efficiency is defined as the probability that a pe will be collected by the dynode structure and multiplied. Collection efficiency is affected by the electric field generated in the PM and the field can be tuned to maximize the collection of pe's. The two quantities qe and ce taken as a product, give a measure of the overall tube response. Monte Carlo detector simulation programs for the IMB detector (to be discussed in a future paper) make assumptions about the shape and magnitude of the qe curve and adjust the ce to fit the Monte Carlo generated data to actual data (ce is nearly independent of light frequency). It is much easier to get a measure of PM relative efficiency ( $\varepsilon$, the counting efficiency or efficiency of getting a signal from an incident photon at a given operating condition; $\varepsilon$ is proportional to qe $\times$ ce), and in fact, this is done regularly in the normal detector calibration procedure. Absolute calibrations of the detector require using cosmic rays (either stopping or through-going).

The venetian blind dynode structure is partially detailed in fig. 1. It is an 11-stage structure of nickel slats coated with a cesium and antimony mixture to enhance their sensitivity to electrons. Each dynode is capable of emitting a few electrons for each incident electron. The number emitted is a function of the incident electron energy (i.e., the voltage between dynodes) and the work function of the dynode material. The number of electrons collected to the next stage however depends upon the field strength and varies significantly with position.

In an ideal photomultiplier, $m$ electrons are produced at the first dynode, and the number of electrons after $n$ stages is $m^{n}$. The output pulse has an amplitude of:

$V=e m^{n} / C$,

where $e$ is the electronic charge and $C$ is the output capacitance. Typical inter-dynode potentials of about $100 \mathrm{~V}$ are useful. Higher potentials increase gain at a less than linear rate with voltage, but phototube noise goes up rapidly probably due to light and ion generation in the lower stages. In this way, a signal of one or more pe is amplified to a signal of a few millivolts, with typical pulse widths of about $20 \mathrm{~ns}$ fwhm into $50 \Omega$, giving about $0.4 \mathrm{pC}$ of charge per $\mathrm{mV}$. In reality, the number of secondary electrons emitted from the dynode fluctuates around a mean value and so PM output pulses vary in amplitude for a given input; giving rise to a distribution of pulse heights. Various people have approximated this convoluted distribution as the Polya distribution, but in fact this mathematical model is faulty and does not describe the observed long tail at large pulse heights seen in the data.

The venetian blind structure, although effective as an electron multiplier, introduces an uncertainty in the time and pulse height response of the PM. This uncertainty is about 2 ns in time and $10-30 \%$ in pulse height for single photon interactions [8]. Measurements show that PM time jitter at one pe illumination is asymmetrically distributed with a fwhm of 10-11 ns (4-5 ns standard deviation about the peak with a tail extending out to $40 \mathrm{~ns}$ beyond the peak). The long electron path length between the pc and first dynode (D1) in hemispherical tubes dominates the increased time jitter. Since electrons are emitted with very little kinetic energy, a relatively long time is taken accelerating the electron the first few centimeters away from the pc. A large potential difference between the pc and D1 is necessary to quickly accelerate pe's and minimize differences in trajectory transit time.

\section{Focusing}

Coupling the dynode structure to the pc is a series of focusing electrodes (shown in detail in fig. 1), which are typically set at potentials larger than the inter-dynode potentials and provide the necessary acceleration and field shape to insure good collection efficiency.

A feature of the PMs used in the detector is their independent focusing electrodes. Operating characteristics, such as time jitter and collection efficiency (which also affect dark noise and afterpulse rates), are affected by the electrode voltage settings. This is shown in tables 1a-1e. The tables list the results of tests used to determine optimum operating voltage settings for the $5^{\prime \prime}$ PM. Tube parameters such as time jitter, $\varepsilon$, dark noise, and afterpulse rate were measured for the PM as a function of $F 1, F 2$, and $D 1$ potentials where $F 1, F 2$, and D1 are labeled in fig. 1. F2 was set to $550 \mathrm{~V}$ in the tests (all voltages are with respect to the pc at ground potential) and was kept constant while F1 and D1 were varied. F2 is mainly an accelerating electrode and primary field shaper, producing a nearly radial field in the region from F2 to the pc. As such, F2 is set to the maximum voltage considered to be safe to prevent improper tube operation (breakdown, ion feedback). The data shown in the tables is for one tube, but we find negligible variation in the electron optics from tube to tube.

From the tables, the conditions which optimize $\varepsilon$ do not necessarily give the best timing response and vice versa. An operating point was chosen to maximize $\varepsilon$ 
Table 1

PM operating parameters as a function of $F 1$ and D1 voltage settings. $F 2$ was fixed at $550 \mathrm{~V}$ in all measurements.

(a) Noise ( $\mathrm{kHz}$ at $20 \mathrm{mV}$ threshold)

\begin{tabular}{rllllll}
\hline D1 [V] & F1 [V] & & & & \\
\cline { 2 - 7 } & 100 & 200 & 300 & 400 & 500 & 550 \\
\hline 0 & 0.93 & 1.25 & 1.27 & 1.21 & 1.55 & 1.43 \\
100 & 0.55 & 1.19 & 1.21 & 1.27 & 1.30 & 1.21 \\
200 & 0.60 & 0.86 & 1.05 & 1.11 & 1.17 & 1.04 \\
300 & 0.44 & 0.64 & 0.99 & 1.48 & 1.49 & 1.39 \\
400 & 0.50 & 0.72 & 0.77 & - & 1.66 & 1.64 \\
500 & 0.47 & 0.62 & 0.74 & 0.83 & 1.23 & 1.58 \\
550 & 0.38 & 0.67 & 0.71 & 0.79 & 0.96 & 1.20 \\
\hline
\end{tabular}

(b) Time jitter fwhm (ns)

\begin{tabular}{|c|c|c|c|c|c|c|}
\hline \multirow[t]{2}{*}{ D1 [V] } & \multicolumn{6}{|c|}{$\mathrm{F} 1[\mathrm{~V}]$} \\
\hline & 100 & 200 & 300 & 400 & 500 & 550 \\
\hline 0 & 12 & 11 & 11 & 11 & 8 & 11 \\
\hline 100 & 12 & 14 & 11 & 10 & 9 & 11 \\
\hline 200 & 9 & 10 & 14 & 10 & 9 & 9 \\
\hline 300 & 8 & 8 & 9 & 11 & 10 & 10 \\
\hline 400 & 10 & 9 & 9 & 9 & 10 & 10 \\
\hline 500 & 8 & 9 & 9 & 8 & 14 & 13 \\
\hline 550 & 9 & 9 & 8 & 8 & 9 & 15 \\
\hline
\end{tabular}

(c) Counting efficiency (arbitrary units)

\begin{tabular}{rllllll}
\hline D1 [V] & F1 [V] & \multicolumn{1}{l}{} & & \\
\cline { 2 - 6 } & 100 & 200 & 300 & 400 & 500 & 550 \\
\hline 0 & 0.65 & 1.10 & 1.31 & 1.42 & 1.61 & 1.61 \\
100 & 0.44 & 1.70 & 1.62 & 1.88 & 2.04 & 1.91 \\
200 & 0.44 & 0.84 & 1.94 & 2.38 & 2.37 & 2.09 \\
300 & 0.37 & 0.65 & 1.19 & 2.62 & 2.56 & 2.45 \\
400 & 0.47 & 0.81 & 0.90 & - & 2.94 & 2.96 \\
500 & 0.35 & 0.65 & 0.83 & 1.07 & 2.02 & 2.64 \\
550 & 0.27 & 0.75 & 0.79 & 0.89 & 1.27 & 1.79 \\
\hline
\end{tabular}

(d) Afterpulse rate $(\%)$

\begin{tabular}{rllllll}
\hline D1 [V] & F1 [V] & & & & \\
\cline { 2 - 6 } & 100 & 200 & 300 & 400 & 500 & 550 \\
\hline 0 & - & 5.4 & 3.5 & 4.4 & 3.9 & 3.8 \\
100 & 0.1 & 5.5 & 4.6 & 5.2 & 4.9 & 3.3 \\
200 & - & 1.2 & 0.7 & 2.4 & 3.9 & 3.2 \\
300 & 0.2 & - & 1.6 & 1.8 & 2.3 & 2.8 \\
400 & 0.2 & 0.8 & 0.7 & 1.7 & 3.6 & 4.1 \\
500 & 0.4 & 0.0 & 0.1 & 0.5 & 0.6 & 4.2 \\
550 & - & 0.7 & 0.6 & 1.5 & 1.9 & 0.7 \\
\hline
\end{tabular}

\begin{tabular}{lllllll}
\hline \multicolumn{2}{l}{ (e) Operating voltage (V) } \\
\hline D1 [V] & F1 [V] & & & & \\
\cline { 2 - 6 } & 100 & 200 & 300 & 400 & 500 & 550 \\
\hline 0 & 2150 & 2150 & 2200 & 2200 & 2350 & 2350 \\
100 & 2350 & 2050 & 2050 & 2150 & 2250 & 2250 \\
200 & 2400 & 2350 & 2000 & 2100 & 2200 & 2150 \\
300 & 2300 & 2250 & 2350 & 2050 & 2150 & 2150 \\
400 & 2350 & 2400 & 2250 & 2350 & 2100 & 2150 \\
500 & 2350 & 2200 & 2300 & 2300 & 2250 & 2200 \\
550 & 2150 & 2350 & 2300 & 2300 & 2400 & 2250 \\
\hline
\end{tabular}


within the constraints of minimum time jitter. Other criteria, not shown, are the shape of the pulse height and timing distributions, the stability of operation of the tube as seen on an oscillope, and computer studies of the electron optics. Consideration of the tables alone would cause a choice of $F 2=550 \mathrm{~V}, \mathrm{D} 1=400 \mathrm{~V}, \mathrm{~F} 1=$ $500 \mathrm{~V}$. Stability of operation and minimum time jitter caused us to back off to $F 2=D 1=550 \mathrm{~V}$, and $F 1=450$ V.

\section{The IMB PM test facility}

As a result of the initial testing of PMs it became clear that, due to variations in tube operating parameters, a large scale testing procedure would need to be developed to select acceptable tubes and grade them by operating voltage for the IMB detector. The most important parameters to measure for PMs in the detector were determined to be: operating voltage to give, in the mean, $50 \mathrm{mV}$ output for one pe input into $75 \Omega$ (an indirect measure of PM gain), dark noise and afterpulse rates, time jitter and pulse height distributions.

A PM testing facility was designed and built at Irvine to carry out the measurements of the above parameters in a controlled way [9]. Also, special tests were performed as more experience was gained with the system. Among these were:

1. photomultiplier tube behavior as a function of light amplitude,

2. photocathode qe measurements,

3. effects of adverse conditions on PMs, and

4. side by side tests of $5^{\prime \prime}$ and $8^{\prime \prime}$ PMs.

The test facility was designed to eliminate, as much as possible, biases due to human observation and error. A series of test programs were developed on an LSI-11 mini-computer (Terak); CAMAC and NIM electronics were used for data acquisition and digitization, and a fast, wide band (200-1100 nm, 1 ns pulse width) pulsed nitrogen arc lamp (Optitron NR-10) was used as the light source. Fig. 3 shows plan and section views of the PM test area and the test facility electronics are outlined in figs. 4a, b. A $4 \mathrm{~m}^{3}$ tank was set up with a cover holding up to thirty-two $5^{\prime \prime}$ or 8" PMs (and later, up to eight $20^{\prime \prime}$ PMs). The tank could be operated empty or filled with reverse-osmosis filtered water, but was normally run empty to minimize cosmic ray produced background light ( $\sim 2 \mathrm{kcs}$ per PM).

Tubes were installed in the test tank under red filtered fluorescent and incandescent light to avoid unnecessary stimulation of the photocathode and minimize the dark-adaptation time. In regular testing, PM serial numbers and tank positions were recorded and the dark room sealed, with PMs initially being set to $1500 \mathrm{~V}$ $\mathrm{K}-\mathrm{A}$ (cathode to anode, or overall voltage) to dark-adapt overnight (12-20 h).
All testing was done under computer control and exceptional tubes were singled out for individual scrutiny at a later time. First, a gain measurement, or "plateau curve" was made. Light from the pulser passed through a series of neutral density (ND) and color filters down a collimating light pipe to a front surface mirror, where it was reflected $90^{\circ}$ towards the bottom of the tank. A plastic Fresnel lens with a focal length of about 2" diverged the light onto a 12 " circular area of white diffusing paint (Plasite). All other surfaces in the tank were painted black to prevent reflections, and so the light was scattered isotropically towards the PMs. This was done to insure that all PMs received the same amount of light with full face illumination. The ND filters were necessary to reduce the intensity of the arc lamp to levels well below single photon mean illumination (a level chosen to correspond to a mean firing rate above $20 \mathrm{mV}$ threshold (occupancy) for the $5^{\prime \prime} \mathrm{PM}$ of about six PM pulses per 100 pulser flashes). The color filters were interference type and a $400 \mathrm{~nm}$ wide band $( \pm 40 \mathrm{~nm})$ filter was normally used to tailor the pulser output spectrum to roughly match the spectrum of Cherenkov light in water. Fig. 5 shows the initial pulser
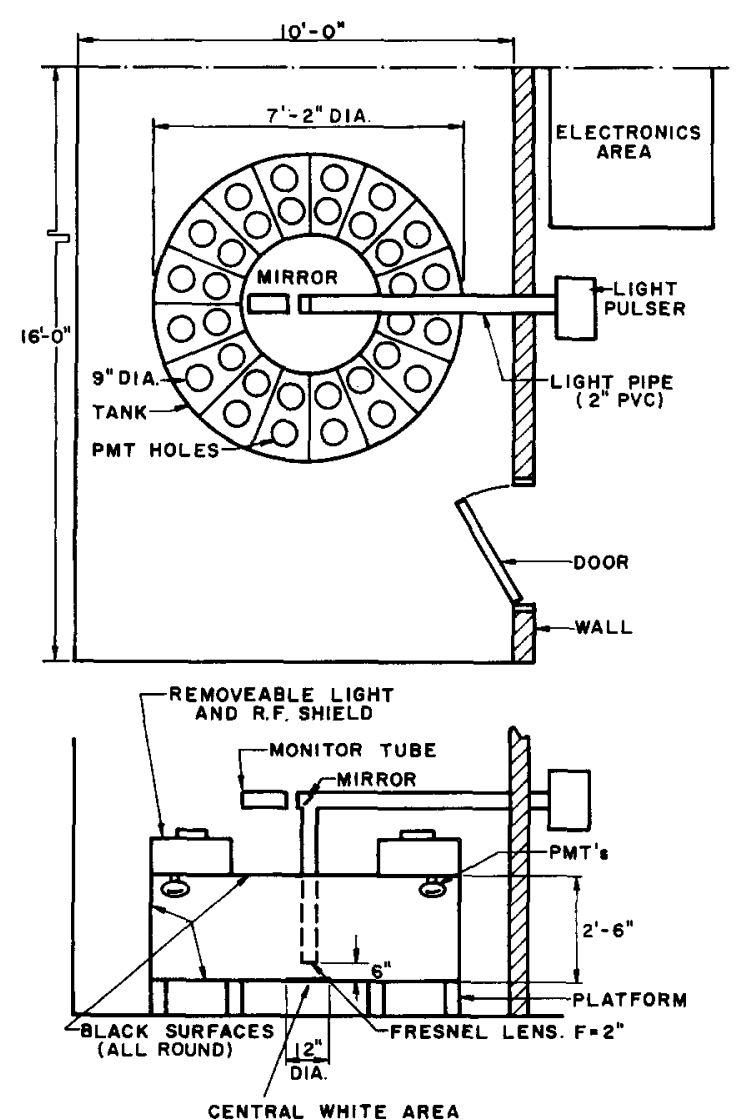

Fig. 3. Plan and section views of the Irvine photomultiplier tube test facility. 

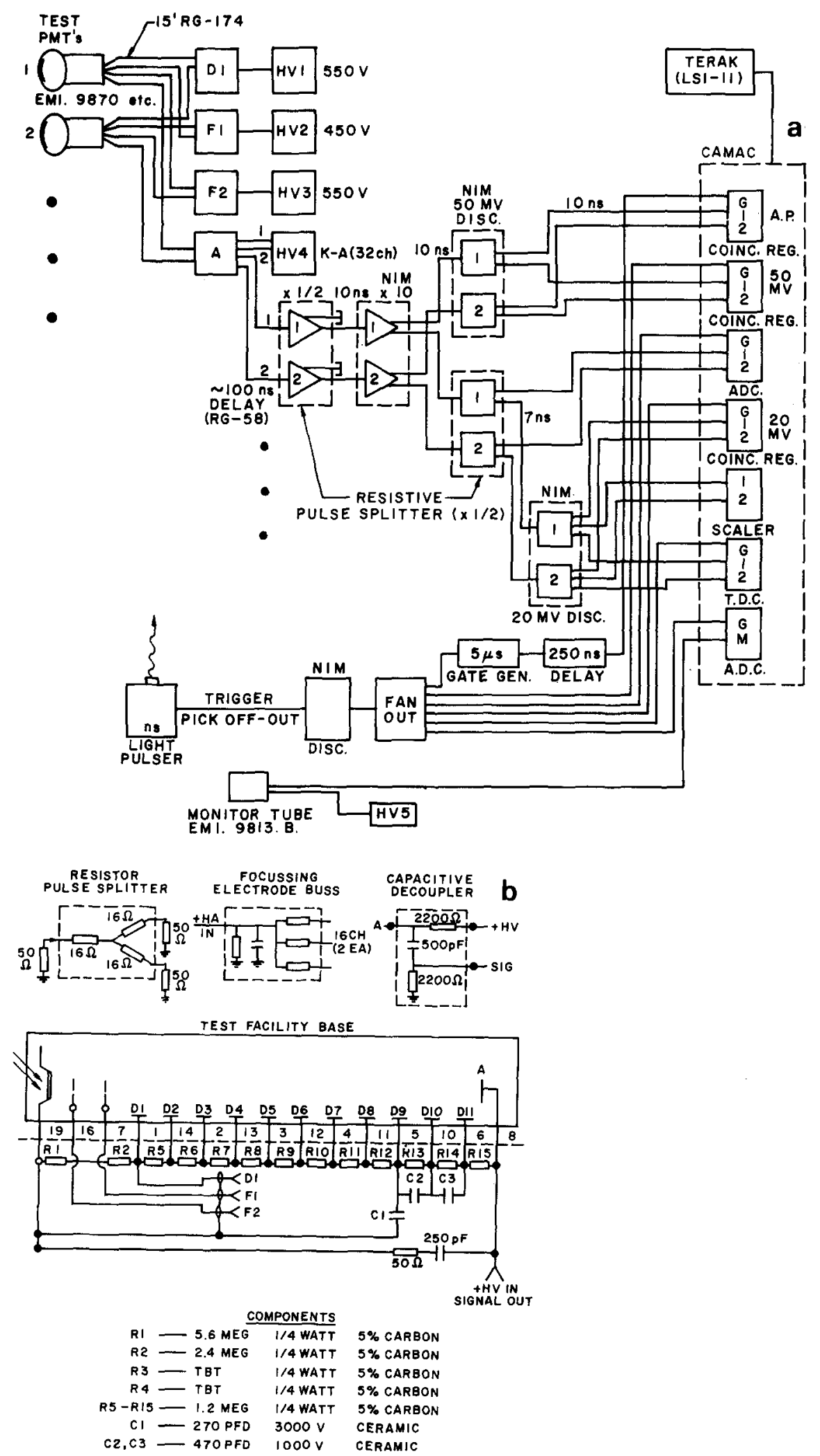

Fig. 4. (a) CAMAC and NIM data acquisition electronics. (b) Test facility $50 \Omega$ PM base with independent focusing capability. 


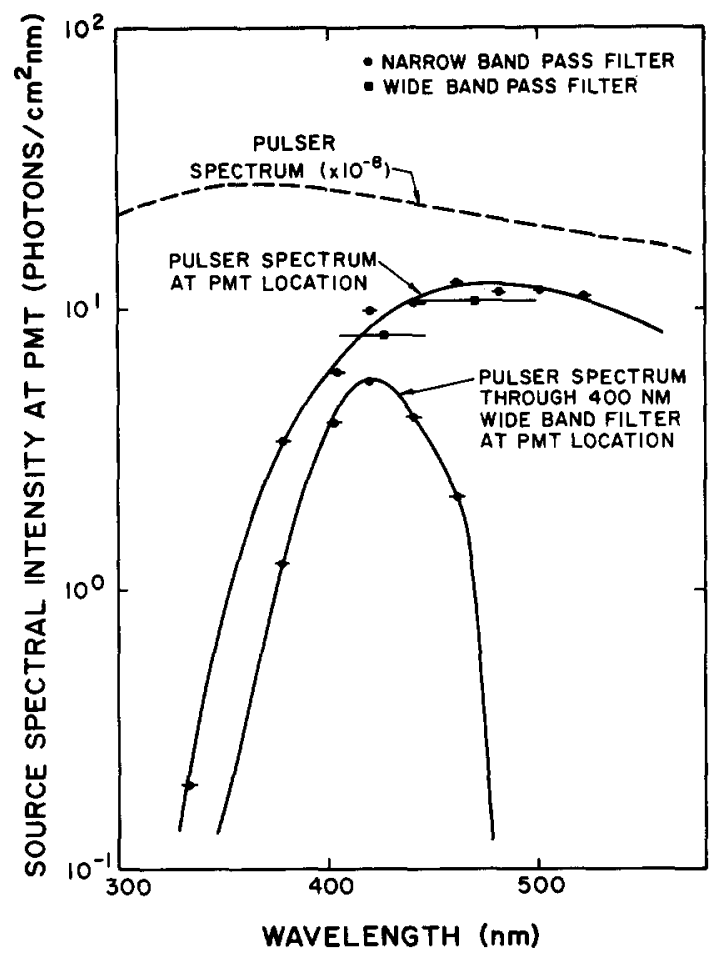

Fig. 5. Optitron spectral intensity measured at Irvine. The spectra are average light output per source pulse.

output spectrum and the spectrum at the PM location. Also included is the pulser spectrum shaped by the 400 $\mathrm{nm}$ wide band filter.

The program initialized each $\mathrm{PM}$ to $1500 \mathrm{~V} \mathrm{~K}-\mathrm{A}$ and raised the voltage in $100 \mathrm{~V}$ increments up to $2500 \mathrm{~V}$ $\mathrm{K}-\mathrm{A}$. Ten thousand pulses were taken at each voltage and the number of pulser-PM coincidences (in a $100 \mathrm{~ns}$ window) at both the $20 \mathrm{mV}$ and $50 \mathrm{mV}$ level were recorded. The data for each tube were plotted as "plateau" or efficiency curves. Each data point was corrected for amplitude jitter in the pulser by using the

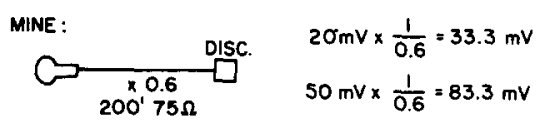

UCI :

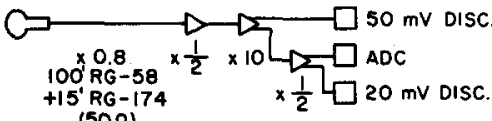

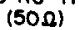

$$
\begin{aligned}
& 20 \mathrm{mV} \times \frac{1}{0.6} \times \frac{50 \Omega}{75 \Omega} \times 0.8 \times \frac{1}{2} \times 10 \times \frac{1}{2}=44.4 \mathrm{mV} \\
& 50 \mathrm{mV} \times \frac{1}{0.6} \times \frac{50 \Omega}{75 \Omega} \times 0.8 \times \frac{1}{2} \times 10 \times \frac{1}{2}=222.2 \mathrm{mV}
\end{aligned}
$$

Fig. 6. Impedance and cable length differences between the Irvine test facility and the mine electronics. The calculations detail discriminator threshold values.
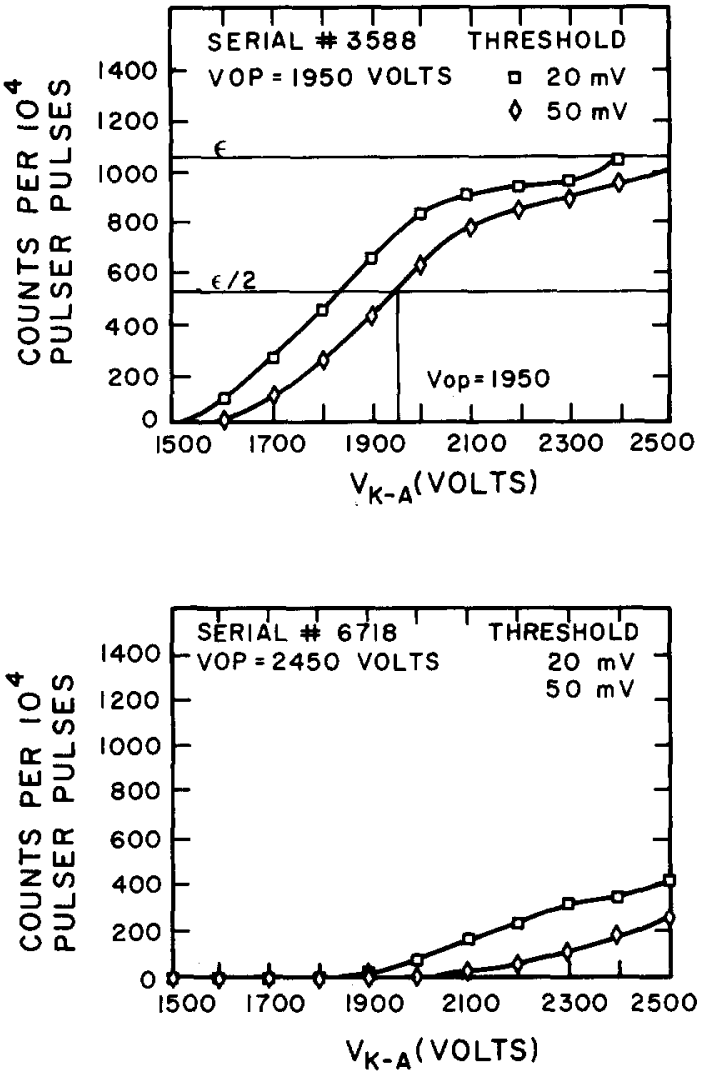

Fig. 7. Typical PM plateau curve.

output of a fast EMI 9813B PM as a monitor for normalization. The 20 and $50 \mathrm{mV}$ discriminator thresholds were "equivalent" thresholds set to take into account transmission losses in the signal cable and impedance differences between the Irvine $(50 \Omega)$ facility and the actual detector electronics $(75 \Omega)$. The corrections are detailed in fig. 6 . The net result was a threshold set to correspond to PM signal amplitudes of 20 and 50 $\mathrm{mV}$ present at the discriminator in the actual detector electronics.

Fig. 7 shows a typical plateau curve for a normal PM. The PM operating voltage (VOP) was set by the computer by taking one half of the maximum $\varepsilon$ on the $20 \mathrm{mV}$ curve $(\varepsilon / 2)$ and finding this value on the $50 \mathrm{mV}$ giving the corresponding voltage, VOP. This is detailed in fig. 7 . If the $50 \mathrm{mV}$ curve never reached $\varepsilon / 2$ the tube was labeled low gain (LG). The algorithm used the fact that the two threshold curves eventually approach the same plateau level at a high voltage (which was usually too high to safely operate the PM). Taking the operating point to be $\varepsilon / 2$ on the $50 \mathrm{mV}$ curve insured that the median pulse height for single pe illumination was 50 $\mathrm{mV}$, i.e., $50 \%$ of the pulse heights would be greater than or equal to $50 \mathrm{mV}$. $20 \mathrm{mV}$ was chosen as the lower threshold to insure that the pm was in the plateau 
region (maximum efficiency) at $2500 \mathrm{~V}$. This was true for most of the tubes tested. Also, rerunning the procedure for the same set of "standard tubes" many times during the course of testing showed that the algorithm reproduced the same VOP to within $\pm 50 \mathrm{~V}$.

Next, the pulser was turned off and a dark noise count rate at the $20 \mathrm{mV}$ level was measured for each $\mathrm{PM}$ at the computer set VOP. Three hundred measurements of $0.1 \mathrm{~s}$ duration were made to calculate the mean noise rate along with the corresponding noise rate jitter. Tubes with large fluctuations in noise were labeled "erratic" and removed from further testing. Tubes with noise rates greater than $32 \mathrm{kcs}$ were labeled "noisy" and also removed from testing. An overall mean noise rate for the detector of less than $3 \mathrm{kcs}$ was established as a design goal and tubes were selected to keep the mean noise under this limit.

Afterpulsing occurs in a PM due to the imperfect vacuum inside the glass envelope. Electron cascades in the dynode from an initial pulse can ionize trace atoms (or knock-out ions from the dynodes), which are accelerated slowly in a direction opposite that of the electrons due to the ion's greater mass and net positive charge. These ions may collide with the photocathode and create a second ("after") pulse occurring typically a few microseconds after the main pulse. Afterpulsing has a time structure correlated to the location of the ionization in the PM and the mass of the ion. Fig. 8 shows a plot of the PM afterpulse spectrum identifying peaks due to protons and helium ions. Note that the delay times of several microseconds are of the same order of magnitude as the muon lifetime $(2.2 \mu \mathrm{s})$. Observation of the muon decay signal in the IMB detector is desirable and so minimizing afterpulsing in the tubes is important.

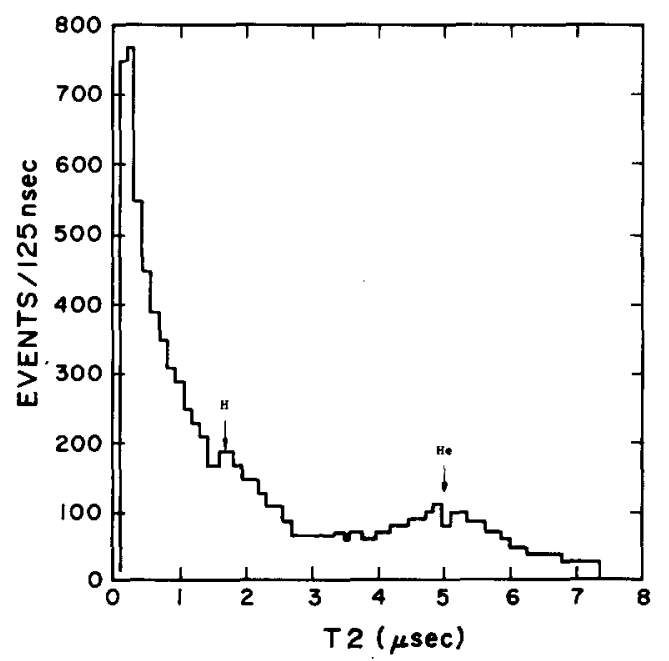

Fig. 8. PM afterpulse time distribution for laser triggers and laser filter 1 . This corresponds to $\gg 1$ pe illumination. At proton decay light levels the rate is much lower.
Afterpulse rates were measured at the $50 \mathrm{mV}$ level (due to the electronics configuration, afterpulse rates could not be measured at the $20 \mathrm{mV}$ level) by using two coincidence registers. One register recorded $50 \mathrm{mV}$ pulser-PM coincidences and the other read $50 \mathrm{mV}$ afterpulses arriving in coincidence with a $5 \mu \mathrm{s}$ gate starting $300 \mathrm{~ns}$ after the pulser trigger. The coincidence registers were read out each time there was a TDC (time to digital converter) interrupt in CAMAC from any one of the tubes. An afterpulse is evident when there are simultaneous hits in the prompt and delayed coincidence registers. The afterpulse probability results were corrected for the effects of random noise during the 5 $\mu$ s gate period.
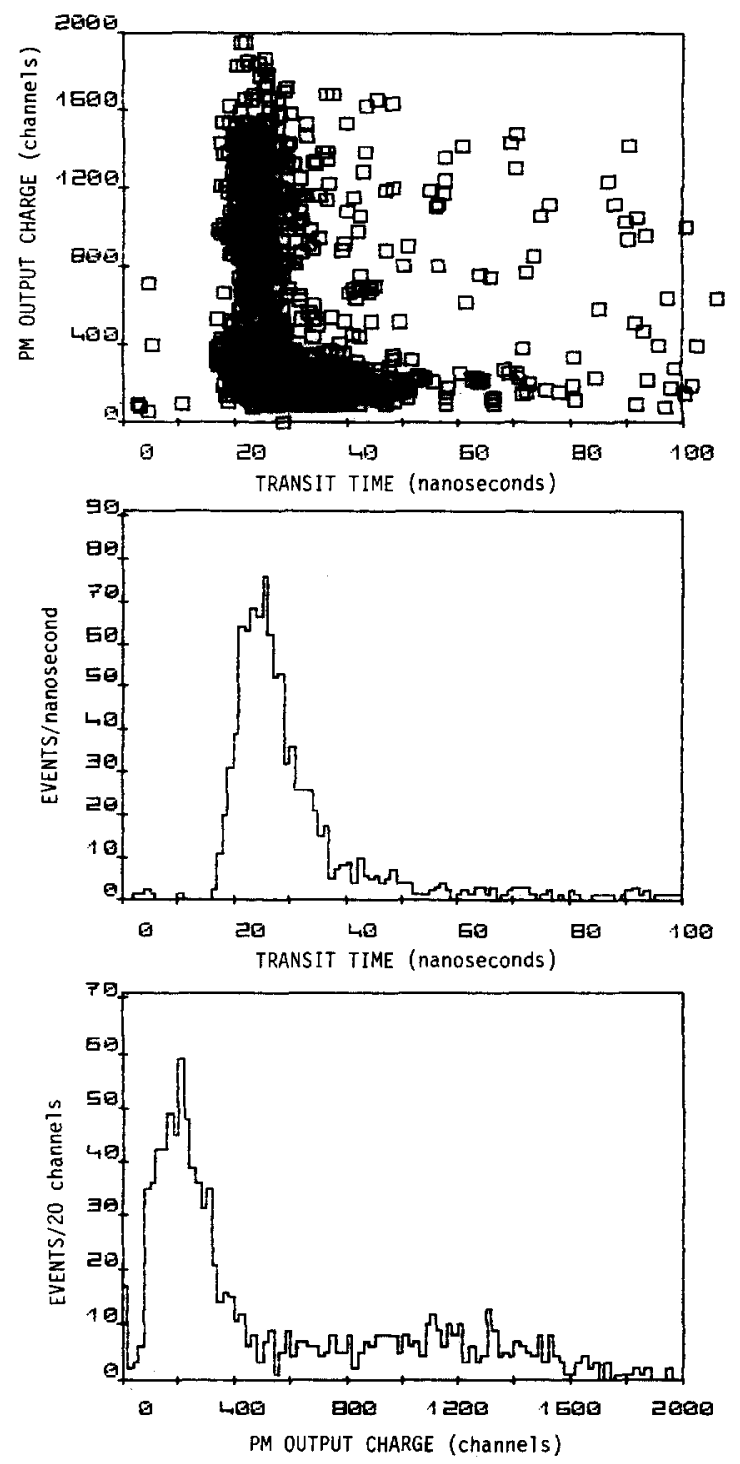

Fig. 9. PM time and pulse height scatterplot with corresponding projections for $\gg 1$ pe illumination. 
Time jitter distributions from each $\mathrm{pm}$ were measured by using a pulse inductively derived from the light pulser breakdown to start a TDC, and PM output signals greater than $20 \mathrm{mV}$ to stop the TDC. There was an optional upper and lower PM pulse height software cut, which could be set at the beginning of the program. Typically, the entire range of pulse heights was used $(0-1980$ bins in the QDC, where one bin equals 0.25 pC). Four thousand data points containing both QDC and TDC information were recorded and histograms of time and pulse height were generated for each tube. Time jitter full width at half-maximum (fwhm) was calculated and a standard deviation was estimated for
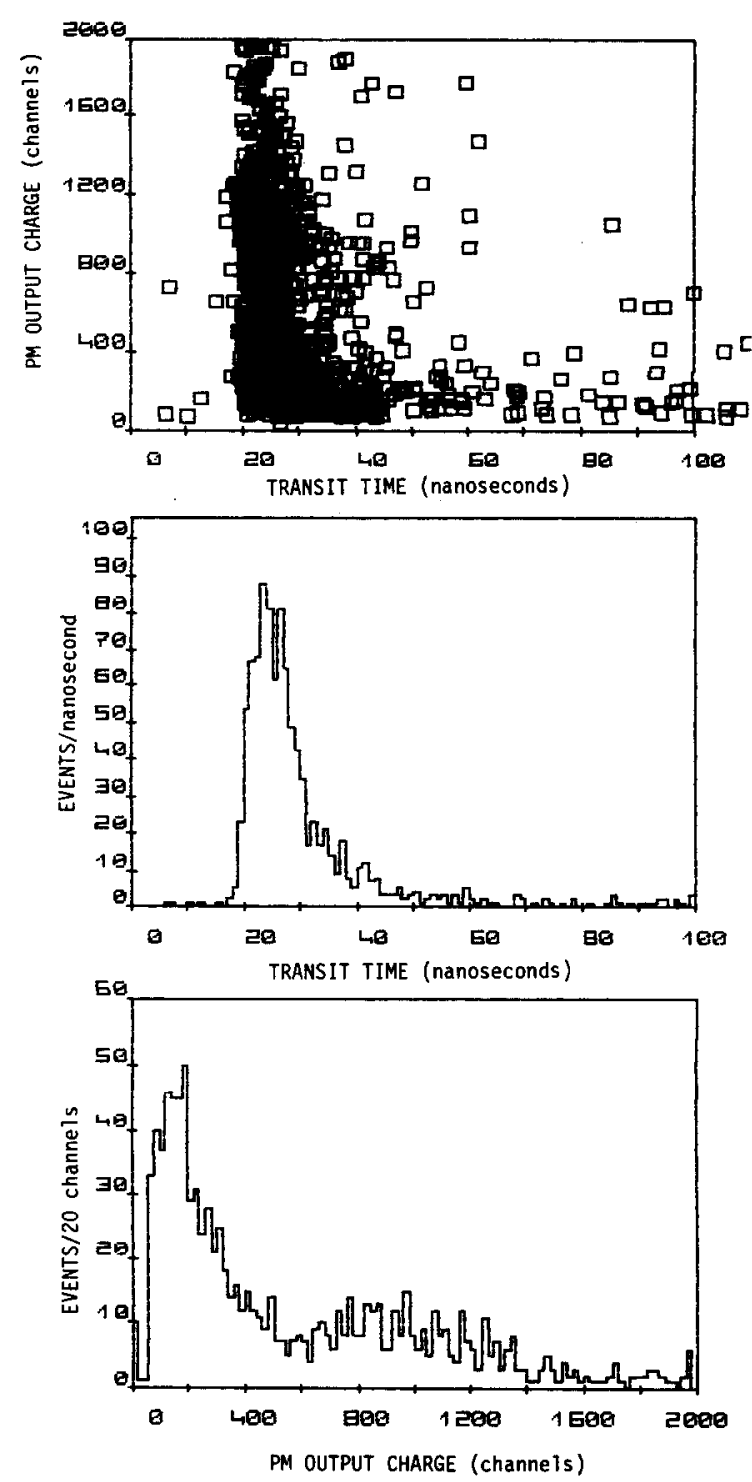

Fig. 10. PM time and pulse height scatterplot with corresponding projections for 1 pe illumination. the time jitter distribution by making a cut on the tail of the distribution.

Since single photoelectron production in a PM gives rise to a distribution of pulse heights, the timing of an output pulse can vary according to the number of secondary electrons generated in the multiplier chain. Each electron has a timing probability distribution associated with it and, for multiple electron generation, the net timing distribution is the product of the individual timing probability distributions. Therefore, PM output pulse time jitter is much greater for low pulse heights than for high pulse heights. Also, the mean transit time of a pulse decreases as the pulse height increases be-
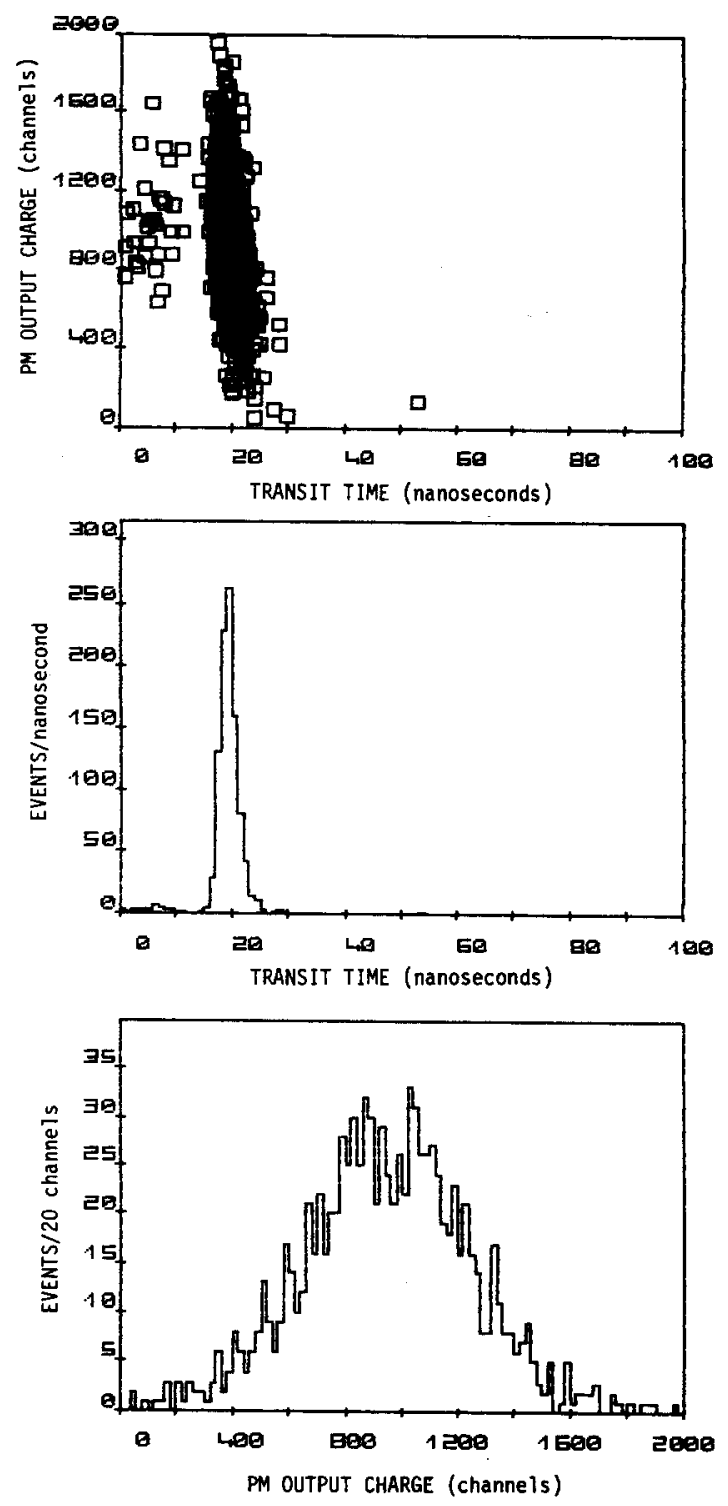

Fig. 11. PM time and pulse height scatterplot with corresponding projections for 10 pe illumination. Note onset of prepulsing in time plot. 
cause the probability of favoring more optimal electron trajectories is enhanced. Finally, the nonzero discriminator threshold causes the mean time of firing to be a function of the steepness of the falling edge of the PM pulse, i.e., small pulses will fire the discriminator later than large pulses. These three properties of photomultipliers contribute to the time slewing effect.

Scatterplots of time versus pulse height can be generated and are useful in studying time slewing and other PM characteristics. Figs. 9-13 show examples of such plots. Note the onset of early pulses for high light levels. These pulses are probably due to photons directly
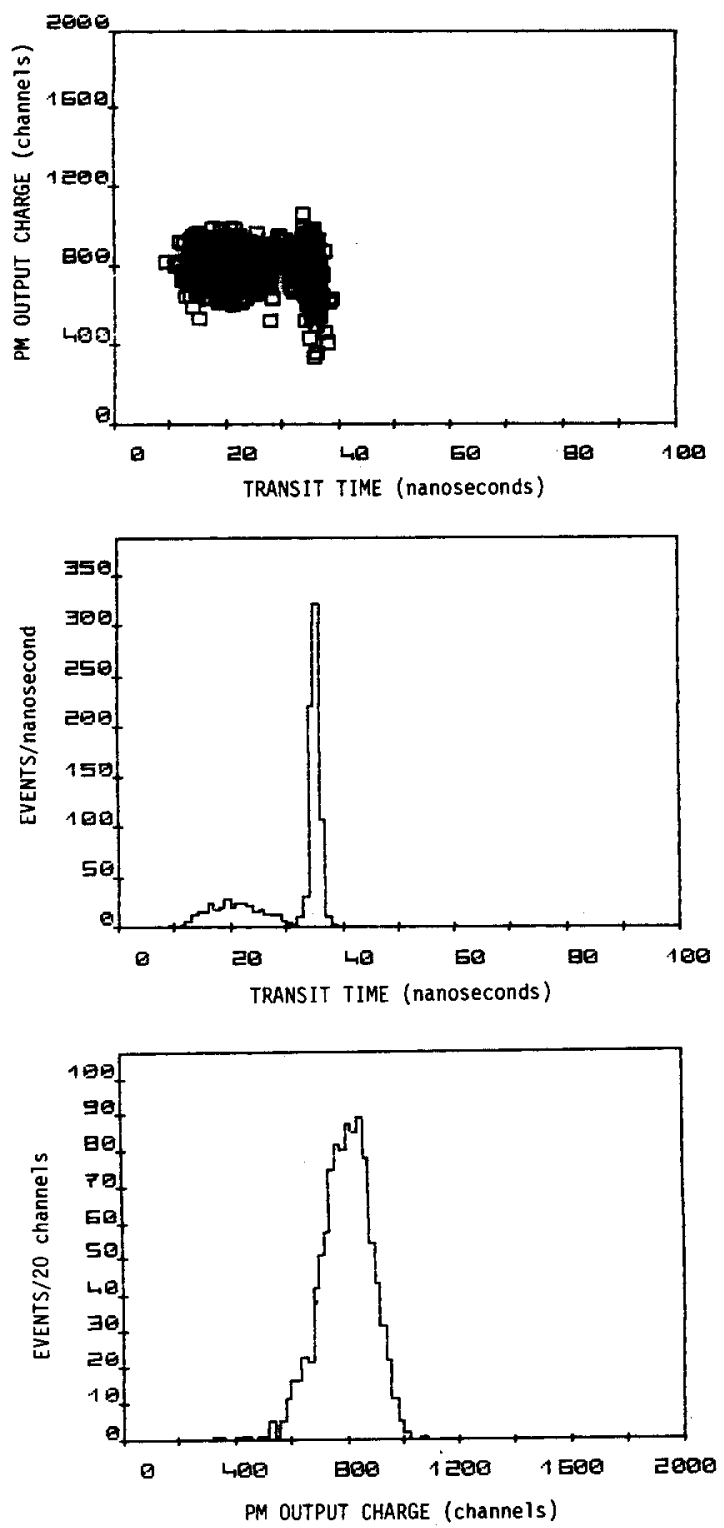

Fig. 12. PM scatterplot and projection for 100 pe illumination (time scale advanced by $15 \mathrm{~ns}$ from previous scatterplots). liberating electrons from the first dynode. The rate is about $0.5 \%$ per pe in these tubes. Also, note the weak second peak in the pulse height distribution. This may be due to electron multiplication on structures in the electron optics, but was present in only about $60 \%$ of the tubes tested. No such peak occurs at higher light levels.

The information from these tests was stored on $8^{\prime \prime}$ floppy disks. Also, hard copies of time jitter histograms, pulse height distributions, noise and afterpulse rates, and plateau curves were generated for all tubes tested. Tubes which passed all of the above tests were labeled
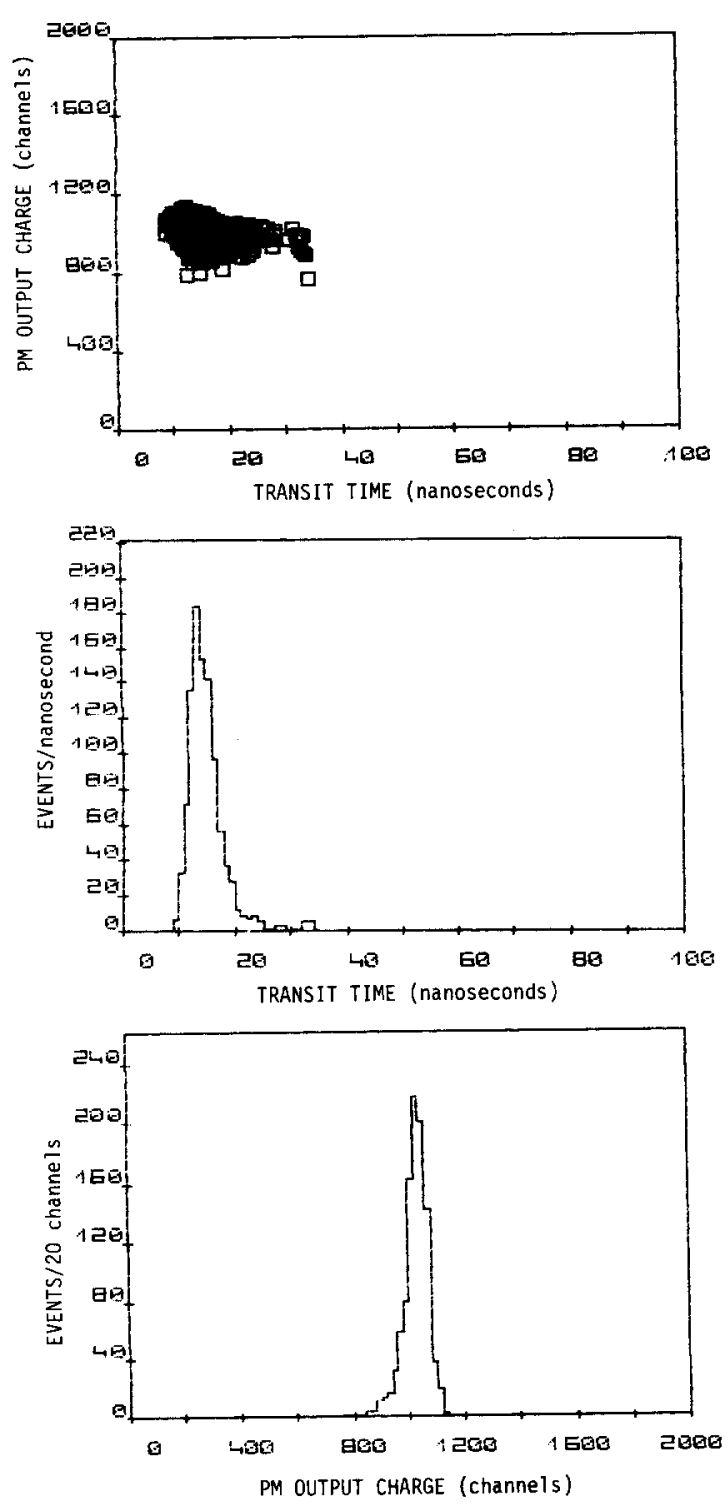

Fig. 13. PM scatterplot and projections for 1000 pe illumination. Signal is almost entirely prepulsing. 
good, marked with their operating voltage, and shipped to the University of Michigan for mounting in PM housings and from there to the salt mine in Cleveland.

\section{Results of PM testing}

Regular production testing at Irvine was limited to 16 tubes per batch for convenience in handling, although the tank could hold up to 32 tubes. The above tests took about two hours to complete. With handling, data logging, and settling time, sixteen tubes per day were easily processed. About 300 PMs per month could be processed by operators working a normal five day week. Regular testing of PMs for the detector was carried out over a 1.5 year period. During that time, 2275 aluminized 5", 100 unaluminized 5", 73 aluminized $8^{\prime \prime}, 70$ unaluminized $8^{\prime \prime}$, and 10 aluminized $20^{\prime \prime}$ Hamamatsu PMs were tested. Also, small samples of early versions of Hamamatsu 5" and 8" PMs were tested (about three tubes per sample) and found to be unacceptable for use in the detector. (They are greatly improved since.)

Table 2 details the results of tube tests for the IMB detector. 2186 tubes were found to be acceptable out of 2491 tubes tested, or $88 \%$ good. The bad tubes break down as follows: 25 dead, 120 noisy, 40 erratic, and 76 low-gain. No tubes were rejected for afterpulsing. Distributions of tube operating parameters, as measured at Irvine, are given in figs. 14-18. Operating voltages are grouped with $80 \%$ of the tubes within a $300 \mathrm{~V}$ region about the mean ( $2050 \mathrm{~V} \mathrm{~K}-\mathrm{A})$. This is a smaller spread

Table 2

(a) $5^{\prime \prime}$ and 8 " phototube testing summary

\begin{tabular}{lrr}
\hline Number of tubes tested & 2491 & \\
\hline Good tubes & 2186 & $87.8 \%$ \\
Noisy tubes & 120 & $4.8 \%$ \\
Low gain tubes & 76 & $3.1 \%$ \\
Bad tubes & 29 & $1.1 \%$ \\
Dead tubes & 25 & $1.0 \%$ \\
Erratic tubes & 40 & $1.6 \%$ \\
Low gain/noisy tubes & 15 & $0.6 \%$ \\
Broken tubes & 14 & $0.6 \%$ \\
\hline (b) 8" PMT testing summary & & \\
\hline Number of tubes tested & 146 & \\
\hline Good tubes & 107 & $73.7 \%$ \\
Noisy tubes & 1 & $0.7 \%$ \\
Low gain tubes & 26 & $17.8 \%$ \\
Bad tubes & 1 & $0.7 \%$ \\
Dead tubes & 9 & $6.2 \%$ \\
Erratic tubes & 0 & $0.0 \%$ \\
Low gain/noisy tubes & 2 & $1.4 \%$ \\
Broken tubes & 0 & $0.0 \%$ \\
\hline
\end{tabular}

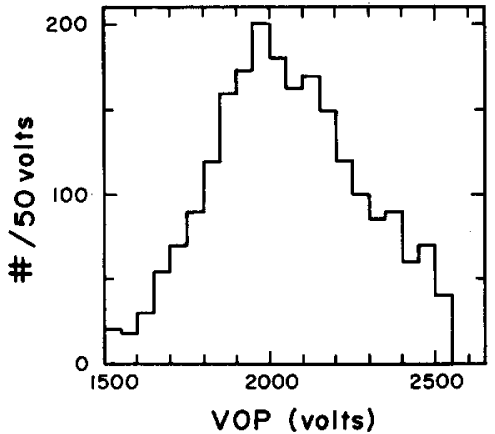

Fig. 14. Summary of PM operating voltages for PMs tested at Irvine.

than was anticipated and allowed for a simpler detector PM base design, using a single base configuration for all PMs, with fixed resistors giving the nominal focusing voltages; $F 2=D 1=550 \mathrm{~V}$ and $F 1=450 \mathrm{~V}$ at $2050 \mathrm{~V}$ $\mathrm{K}-\mathrm{A}$. The distribution of PM mean noise rates is strongly peaked below $2 \mathrm{kcs}$ and has a long exponential tail, with $14 \%$ of the PMs having noise greater than 14 kcs. Tubes with dark noise (measured at a threshold of $20 \mathrm{mV}$ ) of less than $100 \mathrm{cs}$ and greater than one Mcs have been observed. A cut at $32 \mathrm{kcs}$ was made to keep the overall detector average dark noise below $3 \mathrm{kcs}$. We have noted considerable settling of noise rates in the detector in actual operation. After several weeks with no lights on in the detector chamber, the PM noise rate has a median of 1 to $2 \mathrm{kcs}$ per PM. Afterpulse rates have a mean of $2.5 \%$ with $90 \%$ of the PMs having rates less than 6\%. Time jitter fwhm's (uncorrected for pulse height slewing) have a mean of $10.5 \mathrm{~ns} .90 \%$ of the PMs are within $2 \mathrm{~ns}$ of this mean. The relative counting efficiency, $\varepsilon$, is histogrammed on an arbitrary scale from 1 to 10 . The width of this distribution represents both real variations in photocathode efficiency and variations in computer estimates of the plateau height. It is

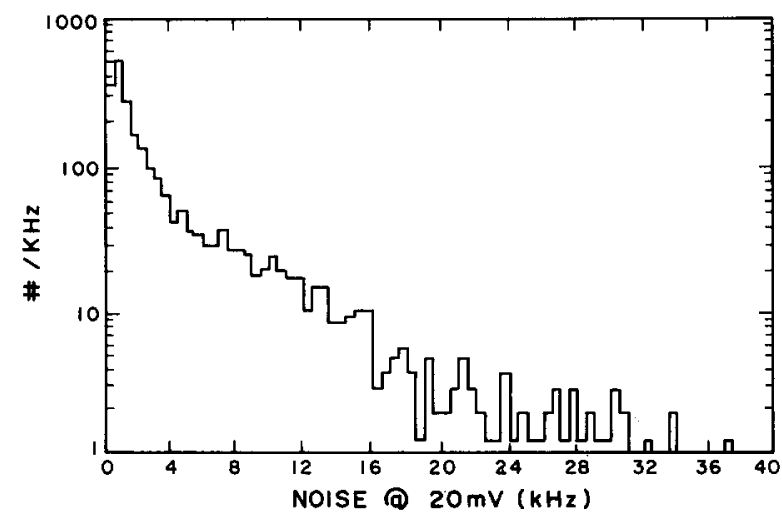

Fig. 15. Summary distribution of PM dark noise rates measured for $20 \mathrm{mV}$ PM output pulses. Note $\log$ scale on the $y$-axis. 
estimated that the plateau algorithm is good to $\pm 10 \%$ and that photocathode sensitivities vary by about $\pm 20 \%$ from the mean efficiency given by EMI. Also, it is assumed that collection efficiency is roughly constant in all tubes tested, due to practically identical focusing configurations in all runs. This allows comparisons of PM quantum times collection efficiency with PM count-

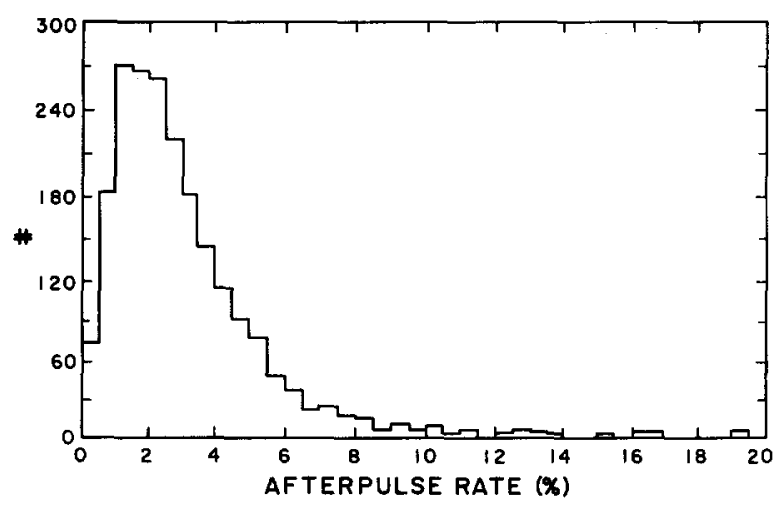

Fig. 16. Summary distribution of PM afterpulse rates measured or PM pulses $50 \mathrm{mV}$.

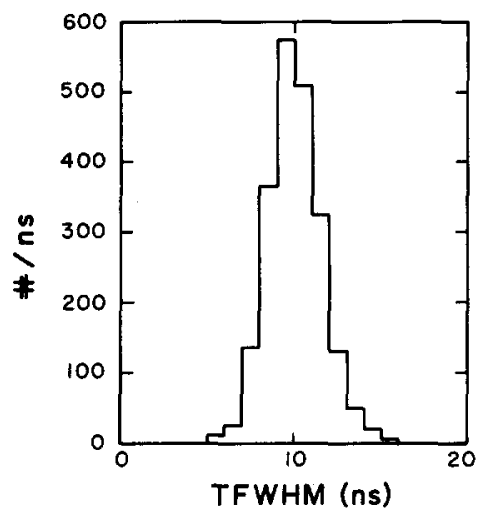

Fig. 17. Summary of PM time jitter fwhm.

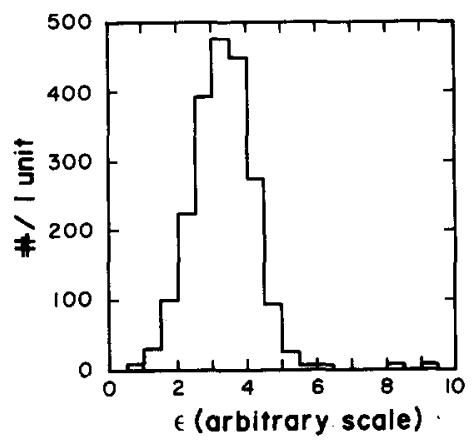

Fig. 18. Summary of PM relative counting efficiency measured at single photoelectron illumination. A PM with an $\varepsilon$ of 4 counts twice the number of pe than at $\varepsilon=2$. ing efficiency (at low light levels $<5$ pe) to give information on the variation of photocathode sensitivities from tube to tube. Figs. 19 and 20 show scatterplots of relative counting efficiency vs Corning blue number and dark current. (Corning blue number and dark current are provided by the manufacturer.)

\section{Time slewing corrections}

Time slewing in PMs was found to be an easily corrected effect. The PM pulse height (QDC) upper and lower cut was varied for a constant 1 pe incident light level, and time jitter histograms were collected. Table 3 details the results of measurements of the mean PM firing time, $T$, and the fwhm and standard deviation of the timing distribution. $T$ is seen to be smaller for higher QDC cuts, and the fwhm and standard deviation tend to be reduced. By fitting a curve of the form:

$\Delta T=A \mathrm{e}^{-B Q}$,

where $A$, and $B$ are constants, $Q$ is the PM pulse height in QDC bins ( $20 \mathrm{mV}$ is roughly 80 bins and $50 \mathrm{mV}$ about 200 bins) and $\Delta T$ is a correction to the measured time $T$ :

$T_{\text {corrected }}=T-\Delta T$.

The time jitter of the PMs can be effectively reduced. Figs. 21a, b show distributions for 27 PMs, corrected and uncorrected. The correction improves the mean transit time jitter of the PMs by $1 \mathrm{~ns}$, from 10.5 to 9.5 ns fwhm. Perhaps most importantly, the slewing correction prevents a serious systematic time shift in the mean for large pulse heights ( $4 \mathrm{~ns}$ at $>100 \mathrm{pe}$ ).

\section{Tube behavior at different light levels}

Tubes were studied at different light levels to gain insight into the PM timing and pulse height behavior. ND filters were used in the study to accurately regulate the light output from the pulser. The ND filters are front surface metal deposited type and are graded according to their optical density (OD) where:

$O D=\log _{10}(1 /$ tr $)$,

and $t r$ is the transmissivity of the filter. Table 4 outlines the conditions for six measurements made at different light levels. Included in the table are ODs and corresponding light attenuation factors, PM trigger rates, mean pe $(\langle n\rangle)$, and relative light levels. Figs. 9-13 show scatterplots of time versus pulse height along with the corresponding projections of time and pulse height. As the light level was increased, both pulse height and time jitter spread were seen to decrease. Also, as the light intensity increased the amount of prepulsing became significant. 


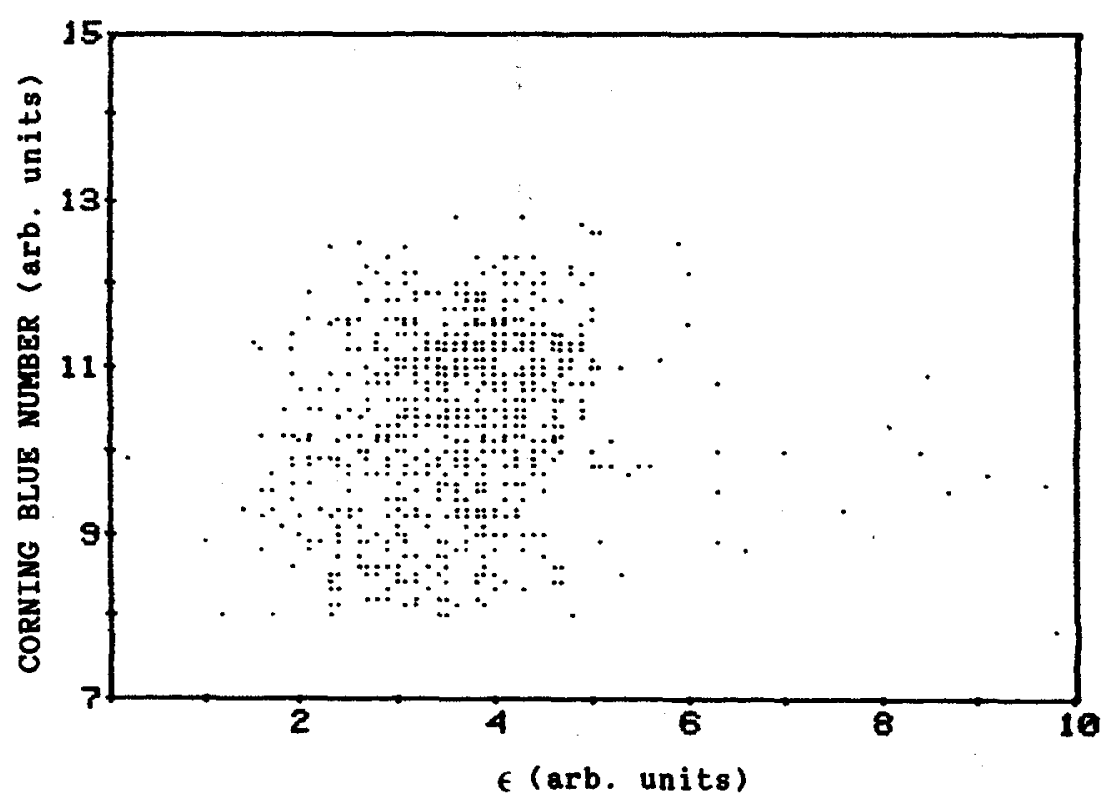

Fig. 19. Scatterplot of manufacturer's measured Corning blue number vs UCI-measured relative collection times quantum efficiency.

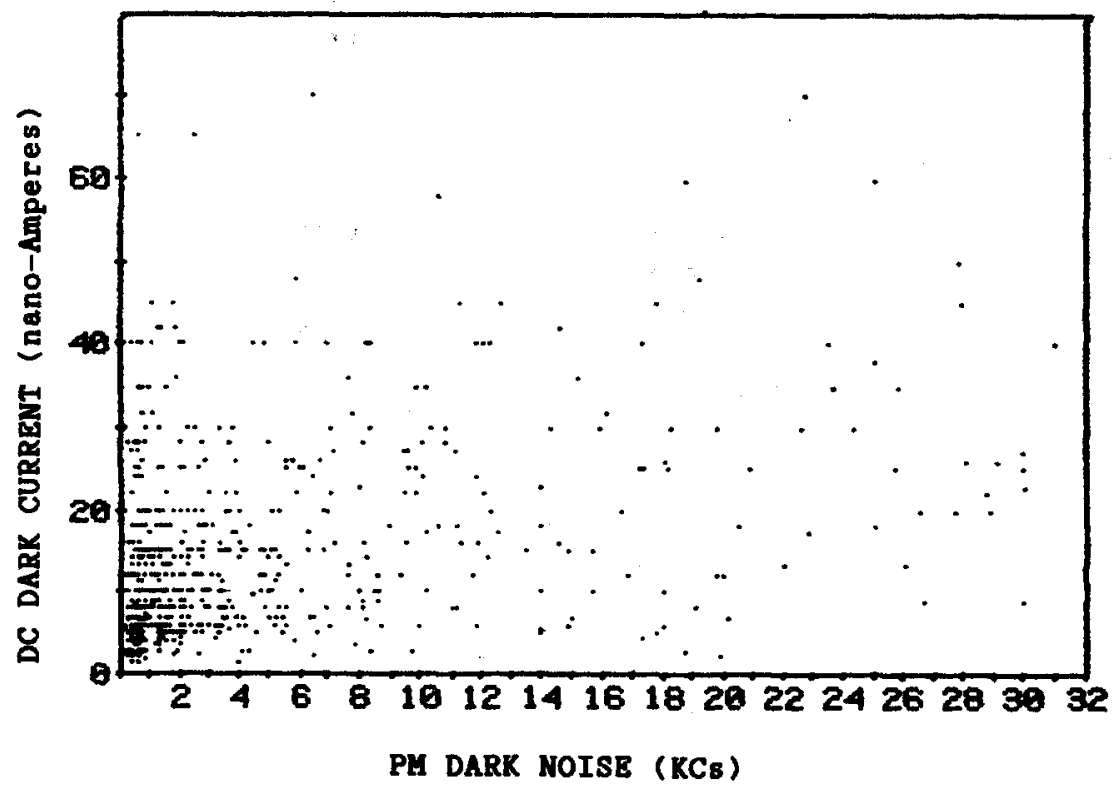

Fig. 20. Manufacturer's de dark current measurement vs UCI PM dark noise measurement at $20 \mathrm{mV}$ and UCI set operating voltage.

Table 3

Results of PM timing measurements as a function of PM output charge. $\sigma_{\mathrm{t}}$ is a standard deviation measured with the tail of the timing distribution cut off. All measurements are in nanoseconds.

\begin{tabular}{|c|c|c|c|c|c|c|c|c|c|}
\hline \multirow[t]{2}{*}{ QDC cut } & \multicolumn{3}{|c|}{ Tube 1} & \multicolumn{3}{|c|}{ Tube 2} & \multicolumn{3}{|c|}{ Tube 3} \\
\hline & $\langle t\rangle$ & $t_{\text {fwhm }}$ & $\sigma_{1}$ & $\langle t\rangle$ & $t_{\text {fwhm }}$ & $\sigma_{t}$ & $\langle t\rangle$ & $t_{\text {fwhm }}$ & $\sigma_{t}$ \\
\hline $100-150$ & 38.6 & 11 & 9.9 & 43.1 & 16 & 9.5 & 39.7 & 10 & 9.3 \\
\hline $150-200$ & 37.7 & 12 & 8.9 & 41.1 & 10 & 8.4 & 38.2 & 11 & 8.5 \\
\hline $200-250$ & 36.6 & 13 & 8.8 & 40.3 & 11 & 8.2 & 37.5 & 9 & 8.5 \\
\hline $250-300$ & 36.7 & 9 & 9.9 & 40.5 & 12 & 8.5 & 37.1 & 10 & 8.4 \\
\hline $300-350$ & 36.5 & 7 & 9.3 & 39.7 & 8 & 7.9 & 37.3 & 9 & 8.6 \\
\hline $350-400$ & 36.4 & 11 & 9.1 & 40.0 & 11 & 8.1 & 36.9 & 10 & 8.6 \\
\hline $400-1800$ & 34.9 & 9 & 9.0 & 38.8 & 9 & 8.0 & 35.9 & 8 & 8.4 \\
\hline
\end{tabular}




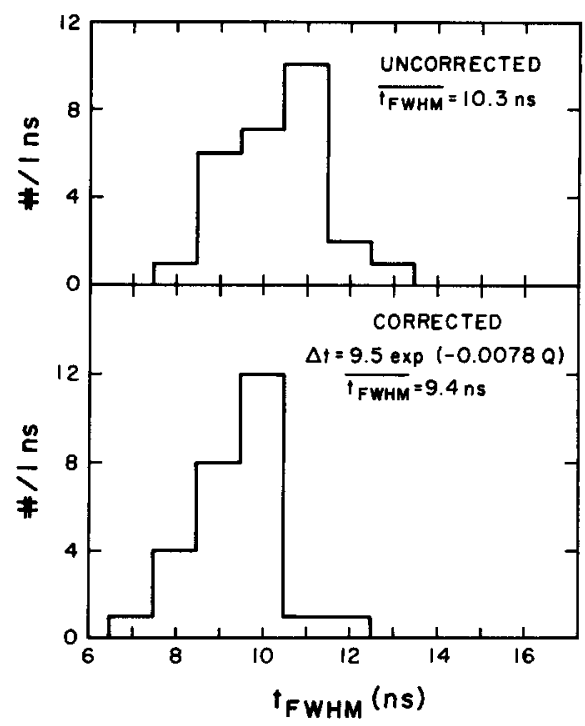

Fig. 21. Effect of time slewing correction on PM time jitter fwhm. A 1 ns improvement in jitter translates roughly into a 22 $\mathrm{cm}$ gain in position resolution.

Prepulsing is the effect of light photons passing through the photocathode surface without interacting, probably striking the first dynode and knocking electrons out. (It occurs at a rate about equal to the relative area of the first dynode to the photocathode, $\sim 1 \%$ in our case.) Prepulsing, therefore, occurs before the regular PM pulse at a time equal to the mean transit time of electrons from the photocathode to the first dynode, about 20 ns for the 5" PMs. As the light level increases, it was also necessary to attenuate the PM output pulse to keep the signal in the range of the QDC. These attenuations are included in the table. At 1000 pe illumination, nearly $99 \%$ of the signal was due to prepulsing. The prepulsing time distribution remained quite broad ( $5 \mathrm{~ns}$ fwhm), even at high light levels where the primary time jitter fwhm is less than $2 \mathrm{~ns}$. This could be due to photons interacting not only with D1, but also with F1 and F2 and knocking off electrons.

Pulse height distributions become narrower and more symmetrical at high light levels. Table 4 gives peak positions and fwhm's for $Q$ and $T$, prepulsing percentage, the time difference between the prepulse peak and the main peak, and some statistical calculations. The expression:

$g=\langle Q\rangle \times 10^{\mathrm{dB} / 20} /(\langle n\rangle \times 500)$,

where the factor 500 is the mean $Q$ at the lowest light level and $\mathrm{dB}$ is the electronic attenuation factor in decibels, gives the relative gain, $g$, of the PM compared to that seen at 1 pe illumination. No drop-off in the value of $g$ as the light level is increased would mean that the PM gain is constant. Fig. 22 shows a plot of $\langle Q\rangle$ versus $\langle n\rangle$ for a typical tube studied in the laboratory. The plot shows that for 1000 times dynamic range in light input the PM has about $\mathbf{4 0}$ times dynamic range in output charge. Note that the cause of this non-linearity is partly due to the PM and partly due to the tube base, being particularly sensitive to the capacitance used in the lower dynode stages.

The calculation of $\langle n\rangle$ at low light levels is based on a statistical method. The method uses the on-off information (occupancy) of the tube to arrive at a light level. Assume the number of pe's is Poisson distributed with

Table 4

Conditions and results for the measurement of PM properties as a function of light intensity.

\begin{tabular}{lllllll}
\hline Run number & 1 & 2 & 3 & 4 & 5 & 6 \\
\hline Optical density & 5.1 & 3.94 & 3.64 & 2.94 & 1.94 & 0.94 \\
Light attenuation factor & 18593 & 8710 & 4365 & 871 & 87.1 & 8.7 \\
Occupancy (\%) & 0.07 & 0.59 & 0.78 & 1.0 & 1.0 & 1.0 \\
$\langle n\rangle$ (pe) & 0.062 & 0.89 & 1.53 & 8.9 & 89.0 & 890.0 \\
Relative intensity & 0.07 & 1.0 & 2.0 & 10.0 & 100.0 & 1000.0 \\
QDC attenuation (dB) & 0 & 2 & 0 & 12 & 22 & 25 \\
$Q_{\text {peak }}$ (ch.) & 200 & 180 & 200 & 890 & 800 & 930 \\
$Q_{\text {fwhm }}$ (ch.) & 200 & 220 & 200 & 700 & 200 & 100 \\
$\langle Q\rangle$ (ch.) & 500 & 600 & 1000 & 890 & 800 & 1030 \\
$t_{\text {fwhm }}$ (ns) & 9 & 9 & 9 & 3 & 2 & $<2$ \\
$\Delta t_{\text {prepulse }}$ (ns) & 20 & 15 & 16 & 13 & 16 & 20 \\
\% prepulse & 0.6 & 0.4 & 0.5 & 4.4 & 24.0 & 99.0 \\
Prepulse probability per pe & - & - & - & 0.005 & 0.003 & 0.005 \\
$Q_{\text {fwhm }} / Q_{\text {peak }}$ & 1.0 & 1.22 & - & 0.79 & 0.25 & 0.11 \\
$2.35 / \sqrt{\langle n\rangle}$ & - & - & - & 0.80 & 0.25 & 0.08 \\
$\langle Q\rangle \times 10^{\mathrm{dB} / 20} /(500\langle n\rangle)$ & 1.0 & 1.20 & - & 0.80 & 0.23 & 0.04 \\
$\langle Q\rangle 10^{\mathrm{dB} / 20}$ & 500 & 600 & 1000 & 3543 & 10071 & 18318
\end{tabular}


mean $\langle n\rangle$ :

$P(n)=\langle n\rangle^{n} \mathrm{e}^{-\langle n\rangle} / n !$

Occupancy (occ) is defined as the probability of one or more pe firing a tube:

occ $=1-P(0)=1-\mathrm{e}^{-\langle n\rangle}$,

so we have:

$\langle n\rangle=-\ln (1-$ occ $)$.

The mean, $\langle n\rangle$, defined in this way, is the mean for all tubes, fired or not, and is thus only useful for single tube measurements. The mean number of pe's for an ensemble of tubes should be taken as the mean number in tubes with one or more pe, because a tube can only give an output signal for one or more pe emitted from the pc. Let $\left\langle n_{1}\right\rangle$ be this latter quantity, then:

$$
\begin{aligned}
\left\langle n_{1}\right\rangle & =\sum_{n=1}^{\infty} n P(n) / \sum_{n=1}^{\infty} P(n) \\
& =\sum_{n=0}^{\infty} n P(n) /(1-P(0)) \\
& =\langle n\rangle /(1-P(0)) \\
& =-\ln (1-\text { occ }) / \text { occ. }
\end{aligned}
$$

This formula is limited in its applicability, but works well at low light levels. The range taken in detector calibration is:

$0.20<$ occ $<0.95$.

Since this calculation works for occupancy less than $100 \%$, an extrapolation of $\left\langle n_{1}\right\rangle$ for occupancy near $100 \%$ is done by using the known increase in transmissivity from the ND filters. The 4 th-6th measurements have $\left\langle n_{1}\right\rangle$ calculated in this way.

Also, calling the probability of a prepulse occurring per pe, $p$, and the observed fraction of prepulses $\alpha$ :

$\alpha=1-(1-p)^{\left\langle n_{1}\right\rangle}$,

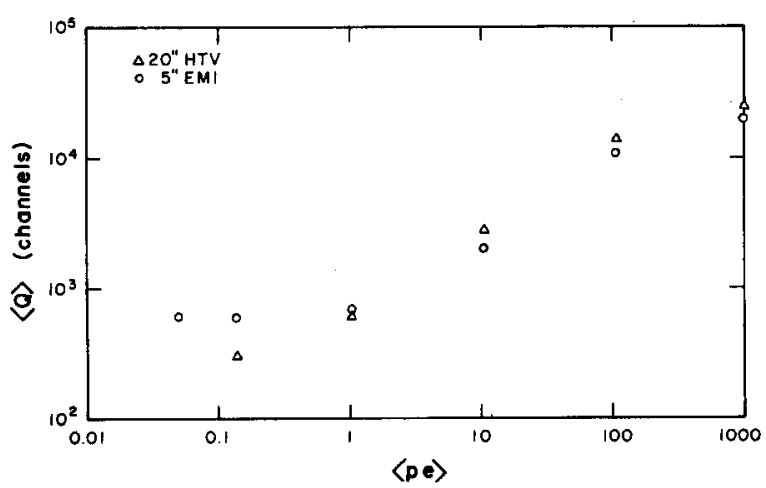

Fig. 22. Average PM output charge as a function of mean number of pe. At $1000 \mathrm{pe}$, the 5" PM rolls off to $1 / 6$ of the linear response vs $1 / 3$ for the $20^{\prime \prime} \mathrm{PM}$. The dynamic range of the $5^{\prime \prime}$ is $\sim 1 / 2$ that of the $20^{\prime \prime}$. for $\langle n\rangle$ mean illumination. This implies that:

$p=1-(1-\alpha)^{1 /\left\langle n_{1}\right\rangle}$.

The prepulse probability, $p$, is calculated for the data in the table and is seen to be approximately constant at 0.005 per pe at all light levels. From the time jitter fwhm information, it is seen that the magnitude of the time jitter is proportional to $\left\langle n_{1}\right\rangle^{-2.5}$.

\section{Tube behavior as a function of light frequency}

Tube operating voltage (gain), $\varepsilon$, timing fwhm, and afterpulse probability has been studied as a function of light frequency by using narrow band width optical interference filters placed in the light beam. As filters were changed, care was taken to adjust the light intensity to insure constant occupancy levels from run to run. The results, averaged over 14 PMs, are plotted in figs. $23 a-c$. Operating voltage remains constant (within an error of $50 \mathrm{~V}$ ), as well as afterpulsing probability, and timing jitter fwhm increases at $330 \mathrm{~nm}$. To verify the effect seen at $330 \mathrm{~nm}$, eight different PMs were measured with 10000 points in the distributions (as opposed to $\mathbf{4 0 0 0}$ points in previous tests). Fig. $23 \mathrm{c}$ shows the result of this measurement overlayed on the first measurement. There is a 1 ns difference between $520 \mathrm{~nm}$ and $330 \mathrm{~nm}$ light, i.e., time jitter is worse for bluer light. A possible explanation is that the higher energy photons

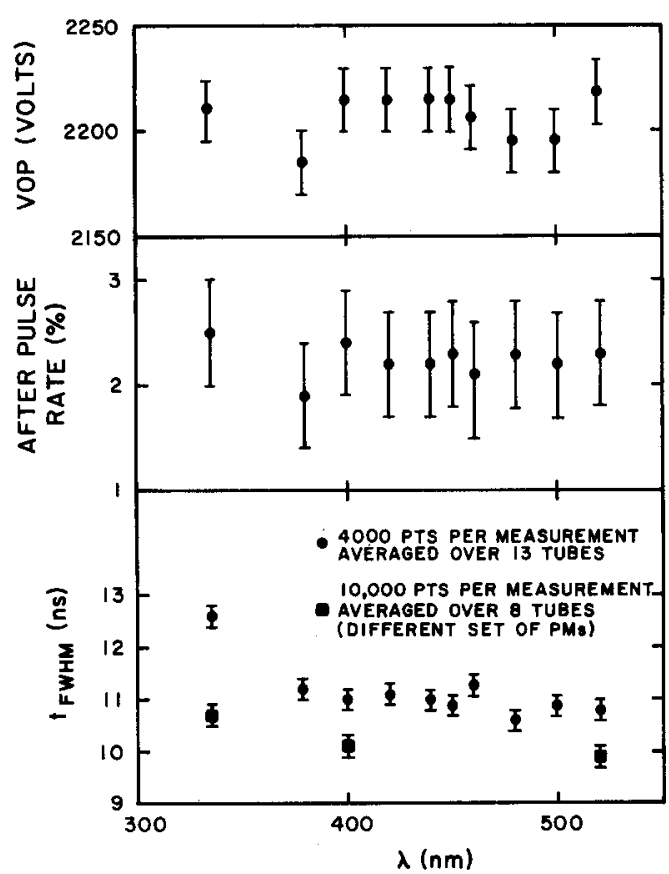

Fig. 23. Measurements of PM operating parameters as a function of light wavelength. Note increase in time jitter at $330 \mathrm{~nm}$. 
interact to knock out pe's with greater transverse momentum and pe trajectories are consequently longer due to this. This result is consistent with the results of ref. [8], who found time jitter to be larger by a factor of 2 for $400 \mathrm{~nm}$ light as opposed to $560 \mathrm{~nm}$ light (measured on an RCA 8850 PM with S1 photocathode).

\section{Measurements of PM absolute quantum efficiency}

A special UV-visible sensitive PIN diode [10] with a response calibrated to a National Bureau of Standards light source was used to measure PM absolute quantum times collection effieciency. The diode response curve is shown in fig. 24. The diode was placed in the test tank near the end of the light pipe to allow a sufficient amount of light to strike its $1 \mathrm{~cm}^{2}$ area and gradually moved away from the light source to measure the intensity as a function of distance. The diode sensitivity is not large enough to allow an accurate determination of the light level at the location of the PM due to its small surface area and an extrapolation of the intensity versus distance relation was used to arrive at the mean light intensity at the PM as a function of wavelength. The spectra shown in fig. 5 were arrived at by using these results.

The PM relative efficiency, $\varepsilon$, can now be corrected to give an absolute qe $\times$ ce. The correction is of the form:

$\mathrm{qe} \times \mathrm{ce}=\{\varepsilon /[(\mathrm{d} N / \mathrm{d} A) A]\} \times 10^{\mathrm{oD}}$,

where $\mathrm{d} N / \mathrm{d} A$ is the intensity of the light source at the PM in photons per $\mathrm{cm}^{2}$, and $A$ is the projected area of the PM: $104 \mathrm{~cm}^{2}$. Fig. 25 shows a result of a calibration for a $5^{\prime \prime}$ EMI and a 20" Hamamatsu PM. The absolute qe $\times$ ce for the $5^{\prime \prime}$ PM is calculated to be $(24 \pm 3) \%$. Assuming $25 \%$ qe for the photocathode (as stated by EMI), the tube ce is seen to be $96 \%(+4 \%,-16 \%)$. The study was done on a "typical" tube and the results should only be taken to show that, as predicted by

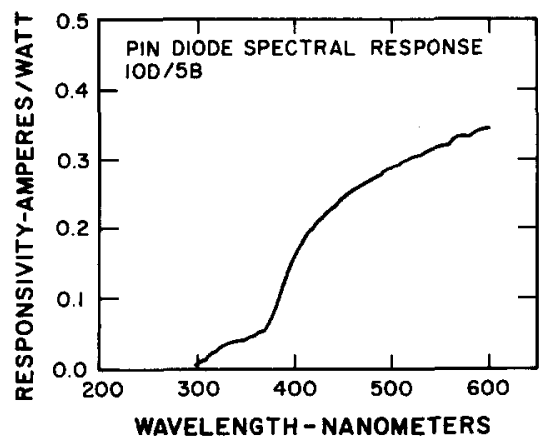

Fig. 24. Response of calibration diode used in PM quantum efficiency measurements.

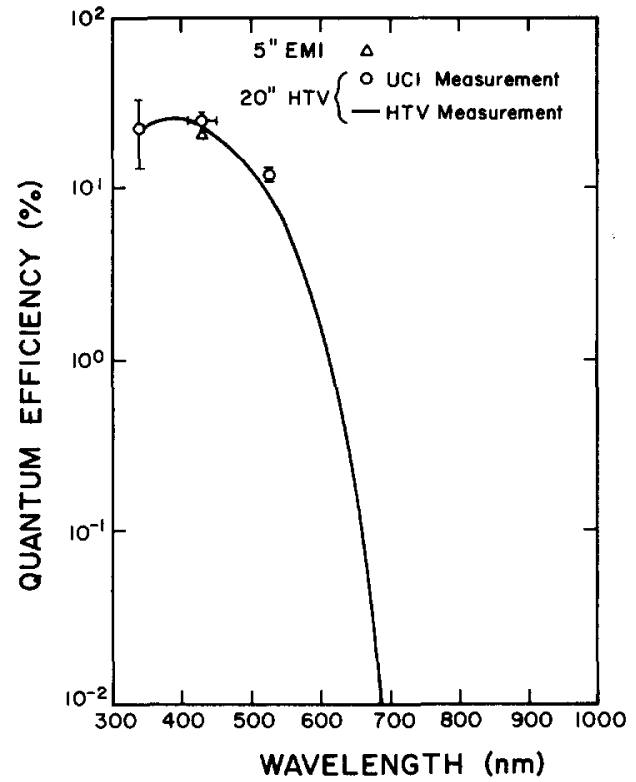

Fig. 25. PM quantum efficiency measurements for the 5" EMI and $20^{\prime \prime}$ Hamamatsu. The Irvine measurements assume $100 \%$ collection efficiency.

computer simulation of electron optics, the collection efficiency is high, certainly much more than $50 \%$ for this tube.

\section{Tube durability and stability}

We have observed that PMs are not absolutely stable devices and are subject to radical changes in operating characteristics. In the final detector configuration PMs fail at the rate of about one per week (out of 2048). Some become excessively noisy or give no output signal. The most common cause of failure appears to be cracks developing in the base of the tube near the pins. As the crack grows, the PM becomes gassy and are observed to emit light which can be seen by other PMs.

Light emitting tubes (LET) were studied at Irvine. Tests were made to study the effect LETs have on normal PMs place nearby. One LET observed actually emitted visible light (observable with the naked eye) of a bluish color. The light is generated, typically, near the anode and surrounds the dynode structure in a bluish haze. It is thought that these are gassy tubes, although no loss of vacuum is observed (i.e., the photocathode appears normal; PMs with gross cracks lose their photocathode and become clear). Three LETs were found in the 2500 PMs tested. The conclusion is that the most significant effect on nearby PMs due to exposure to LETs is the reduction in cathode efficiency, other tube characteristics appear to be only temporarily affected. From the experience gained in this test, it was seen that 
LETs, when they occur, are easily isolated in the IMB detector. Adjacent tubes have very large noise rates, and plots of PM noise rates give an indication of the LET location. Also, current monitoring of the PMs allow LETs to be spotted since they typically draw a factor of 10 more current than normal. LETs have, in fact, been observed in the detector and, in all cases, have been successfully isolated and removed from the system. The LET phase is typically a step on the road to complete tube failure.

From the above discussions, it is apparent that PMs must be continually monitored (and calibrated). Since the detector has been in operation (as of 1 February 1984 - 1.5 years total), 63 PMs have gone bad for reasons not associated with water leakage in the tube housing or electronics. Thirty four PMs have become noisy possibly due to small amounts of water vapor in the PM housing. On the other hand, regular maintenance on bad PMs has reduced the number of PMs out of service from ten per week to about one per week over the last twelve months of operation (about $18 \times 10^{6}$ PM-hours).

\section{2. $P M$ performance}

This section will describe PM operation and performance in the IMB detector as a check on the test procedures detailed previously. Tubes are operated under slightly different conditions in the IMB detector than they were at the Irvine PM test facility. First, the PM is equipped with a fixed resistor base, shown in fig. 26 , which is designed to be as simple as possible for reliability and high impedance to provide low current consumption. Second, the digitization electronics is set up with a single discriminator, one QDC and two TDCs per tube. The discriminator is computer adjustable down to about $15 \mathrm{mV}$ (into $75 \Omega$ ), the two TDCs are a 0-512 $\mathrm{ns}, 1 \mathrm{~ns}$ least count fine time clock and a $0-7.5 \mu \mathrm{s}, 15 \mathrm{~ns}$ least count course time clock, and the QDC is a $0-512$ count, $1 \mathrm{pC}$ per count charge digitizer. Time jitter and pulse height distributions for PMs in the detector can be obtained utilizing a pulsed nitrogen laser ( 1 ns pulse width, $337 \mathrm{~nm}$ wavelength) via an optical fiber into a diffusing sphere mounted in the center of the detector. Also, ND filters are available for relative calibration of PM counting efficiency. A calibration procedure is carried out on a weekly basis to provide the necessary conversion parameters for timing and pulse height. The timing calibrations are done by varying the laser firing time with respect to the system global trigger. Line fits are made to plots of measured PM firing time versus global trigger delay time to arrive at the necessary counts to nanoseconds conversions for each individual tube. Counting statistics can be used at light levels below 5 pe to arrive at a calibration for PM pulse

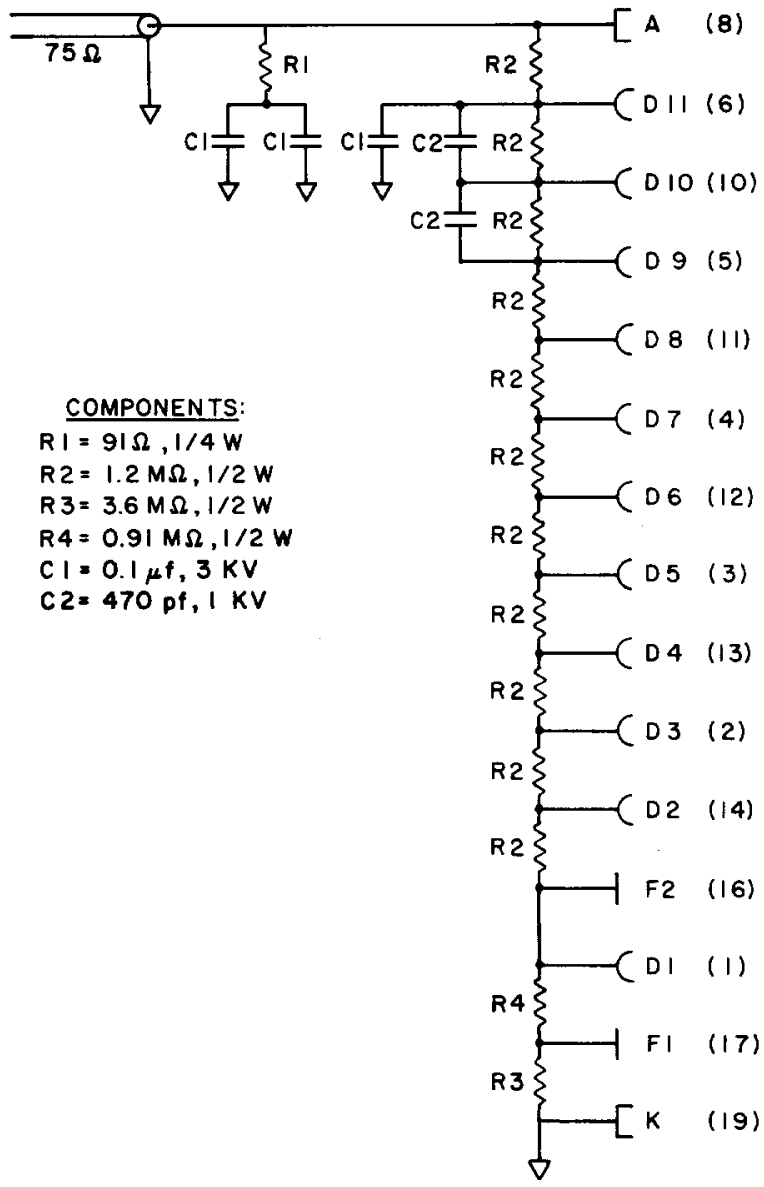

Fig. 26. The IMB PM voltage divider (base). Resistor values are high to allow low current consumptions. In this way, 16 PMs can operate on a single hv channel.

heights. Lines are fit to plots of $n_{1}$ versus $\left(Q-Q_{\text {ped }}\right)$, where $n_{1}$ is the number of photoelectrons defined in eq. (1), $Q$ is the measured pulse height in counts and $Q_{\text {ped }}$ is the measured charge digitizer pedestal, to arrive at a counts to photoelectron conversion slope. With the above calibrations, checks have been made on detector performance by measuring known cosmic ray properties such as angular distributions, stopping to through-going muon ratios, and muon lifetime [11]. Also the laser diffusing ball position is regularly reconstructed as a check on the resolution of the entire detector. Both of these checks show that the detector does indeed perform as expected. The results show that the mean $x, y$, and $z$ position of the ball are fit to within $10 \mathrm{~cm}$ of the actual laser ball with an uncertainty (standard deviation) of about $20 \mathrm{~cm}$ in all coordinates. This includes not only the effects of PM time jitter, but also errors associated with the calibration procedure and the off-line reconstruction program. Despite the added errors, the position uncertainty is less than one meter at single photo- 


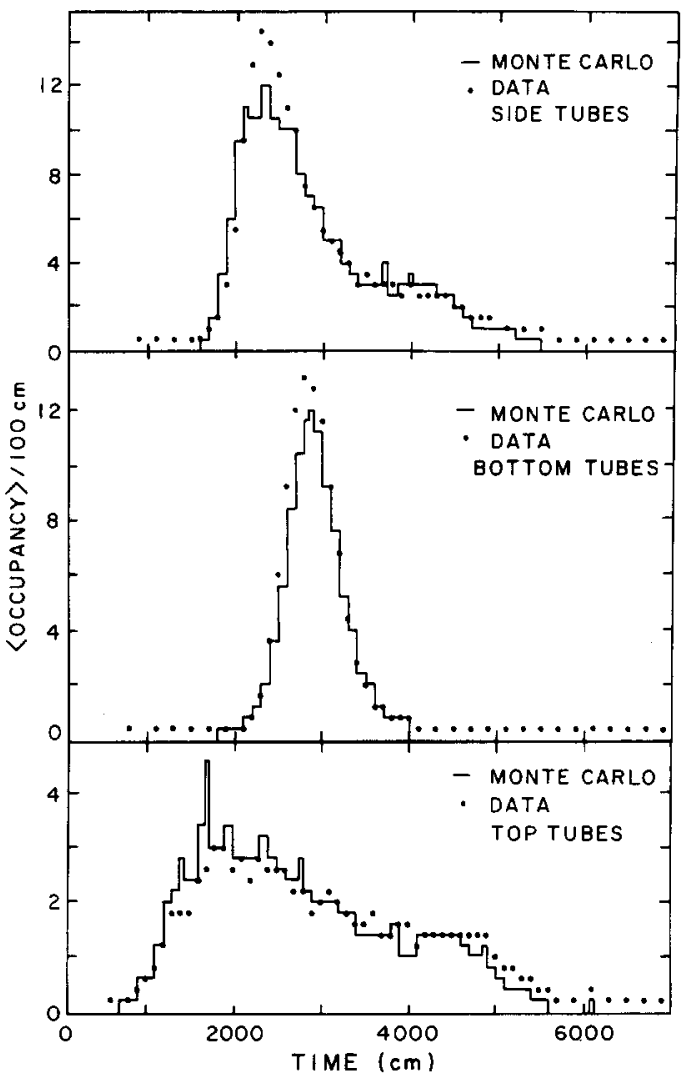

Fig. 27. Comparison of Monte Carlo with data for PM time distributions. The detector is divided into three regions: side tubes on the upper half of the walls, bottom plus side tubes on the lower half of the walls, and top tubes only. This is done to determine the goodness of the fit in different areas where the amount and type of light is different. For example, the top plane of tubes receives light which is mostly scattered. Good fits to the data in this region are important in understanding the effects of Rayleigh scattering.

electron light levels, which is entirely consistent with the distance uncertainty associated with the $5 \mathrm{~ns}$ PM time deviation $(22 \mathrm{~cm} / \mathrm{ns}=$ speed of light in water).

Cosmic ray muons provide a further check on PM performance by allowing absolute measurements of the photon to photoelectron or MeV conversion. Fig. 27 shows the distribution of PM timing in the detector for a sample of near vertical through-going muons along with a computer generated sample of like muons, produced with the effects of additional light generating mechanisms such as brehmsstrahlung, knock-ons, pairproduction and light scattering (Rayleigh). The shape of the data distribution is well matched to the Monte Carlo results which treats the Rayleigh scattering probability and the PM quantum efficiency as free parameters. With this fit, single charged particles are generated
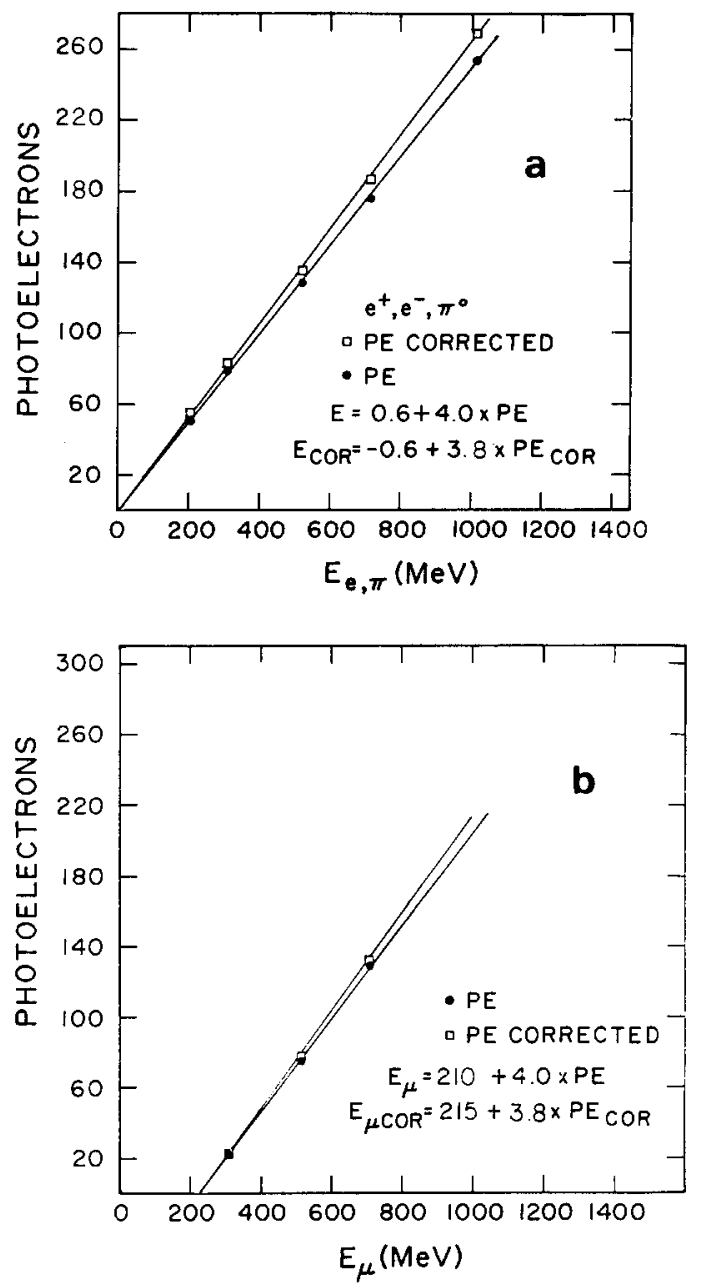

Fig. 28. (a) Monte Carlo results for the conversion of photoelectrons to energy deposited above Cherenkov threshold, in $\mathrm{MeV}$. Neutral pions are essentially identical in energy deposited per photoelectron to electrons. One curve is sufficient to describe both types of particles. (b) Monte Carlo results for the conversion of photoelectrons to energy deposited above Cherenkov threshold, in MeV. Muons have a larger rest mass than electrons and this gives rise to an offset of $215 \mathrm{MeV}$ to the visible energy generated in a muon track. The slope of the curves is identical to those for electrons and neutral pions and so any track attributable to a muon must have its energy increased by $215 \mathrm{MeV}$.

in the simulated detector and energy deposition as function of the number of photoelectrons is calculated. Figs. 28a, b show curves for electrons, neutral pions, and muons. Comparing the computer generated cosmic rays with the data gives an energy calibration of about 4.0 MeV of deposited energy in the detector per photoelectron. These measurements also indicate that the systematic uncertainty is about $15 \%$, which is consistent 
with expectations given the uncertainties associated with the PM electron multiplier at single photoelectron light levels [12].

\section{Conclusions}

We have demonstrated that the large number of photomultiplier tubes necessary for the IMB detector can be processed using a computer controlled test facility of modest scope. We estimate the cost of the test setup to be roughly $\$ 40 \mathrm{k}$ and that PMs can be processed at a cost of about $\$ 0.50$ per unit. After installation the PMs remain unchanged in their operating characteristics $(15 \%$ uncertainty in pulse height, 4-5 ns uncertainty in time, at single pe illumination, and $-2 \mathrm{kcs}$ average dark noise). The large number of PMs coupled with the long running time has provided valuable information on PM durability; namely, in 18 million PM-hours of operation, 96 out of 2048 or $4.6 \%$ of the PMs have failed for reasons not associated with the system. This corresponds to roughly 21 years MTBF for installed tubes.

Finally, we would like to point out that the Irvine PM test facility represents a method of PM testing and classification more relevant to high energy physics experiments involving small numbers of photoelectrons and counting applications than the industry standard measurements. In addition, results are unbiased and the large quantities of data can be stored for future reference.

It is clear from our experience that these PMs outperform many of the manufacturer's claims, in particular with respect to operating voltage and gain. We have found that the quantities traditionally measured by the manufacturer: overall sensitivity $(\mathrm{A} / \mathrm{lm})$, dark current (A), and Corning blue number (a dimensionless measure of the pc blue light response) [13] are not sufficient quantities for specifying tubes for critical counting applications such as the IMB experiment. However, they are undoubtedly useful selection parameters since the overall yield to the IMB specifications turned out to be about $88 \%$, despite the operation of tubes at 10 times the gain they were designed for.

We would like to suggest to the community that new standards of measurement should, and with existing technology could, be implemented that would benefit both producers and physics community consumers. 'As a beginning we suggest the following for overall specifications.

1. Supplementing the Corning blue number with the actual quantum times collection efficiency averaged over the photocathode surface with stated acceleration and focusing voltages.

2. In addition to overall PM sensitivity measured in A $/ \mathrm{lm}$, we proposed a quantity that relates to gain at the one photoelectron level. For example, in our case, we use the voltage that produces a median signal amplitude of $50 \mathrm{mV}$ in a $50 \Omega$ load per photoelectron under single photon illumination.

3. To supplement the manufacturer's dark current measurement, we suggest the dark count rate above some fraction of a single photoelectron equivalent signal (in our case we chose $0.4 \mathrm{pe}$ ).

4. A standard for time resolution poses more of a problem because time distributions are highly skewed and do not, in our experience, fit a simple distribution. We suggest that the fwhm of the time jitter peak and the time window which contains $90 \%$ of the data should be presented for single photoelectron illumination. Perhaps similar values at 100 pe would also be useful.

We would like to thank Thorn EMI Ruislip, UK and Thorn EMI Gencom, New York, and their representatives, for their help and numerous discussions in implementing the Irvine PMT test facility. We would specifically like to acknowledge the help of C. Cory, J. Haskey, and C. Pagels at UCI for programming help and carrying out many of the tests reported herein. Also, we are grateful to the Fairport mine of Morton-Thiokol Company in Painesville, Ohio for allowing us to access to their facilities.

\section{References}

[1] M. Goldhaber et al., Proposal for a Nucleon Decay Detector (May 1979) unpublished.

[2] S.L. Glashow, Nucl. Phys. 22 (1961) 579; S. Weinberg, Phys. Rev. Lett. 19 (1967) 1264;

A. Salam, in: Elementary Particle Theory, ed., N. Svartholm (Almqvist and Wiksell, Stockholm, 1968) pp. 367-377.

[3] G. Arnison et al., Phys. Lett. 122B (1983) 103; Phys. Lett. 126B (1983) 398; Phys. Lett. 129B (1983) 273.

[4] H. Georgi and S.L. Glashow, Phys. Rev. Lett. 32 (1974) 438;

H. Georgi, H.R. Quinn and S. Weinberg, Phys. Rev. Lett. 33 (1974) 451.

[5] R.M. Bionta et al., Phys. Rev. Lett. 51 (1983) 27. See also ref. [12].

[6] Thorn EMI Inc., Phototubes and Accessories Catalog, $10 \mathrm{M} 278$.

[7] M. Jedlicka et al., 3rd Czechoslovak Conf. on Electronics and Vacuum Physics Trans., Prague (1967) p. 351.

[8] M. Moszynski et al., Nucl. Instr. and Meth. 141 (1977) 319.

[9] The UCI PMT test facility was designed and built by C. Cory, J. Haskey, J.G. Learned, D.W. Smith and C. Wuest. A series of memos detailing the work carried out during the testing of photomultiplier tubes are available upon request. Also, a review paper on the PMT test facility appears in C. Cory et al., IEEE Trans. Nucl. Sci. NS-28 (1981) 445 
[10] The PIN diode is a United Detector Technologies PIN. $10 \mathrm{D} / 5 \mathrm{~B}$.

[11] R.M. Bionta et al., Proc. 17th Int. Cosmic Ray Conf., Paris (1981) vol. 7, p. 184.
[12] B.G. Cortez, PhD Thesis, Harvard University (1983); G.W. Foster, PhD Thesis, harvard University (1983); E.L. Shumard, PhD Thesis, University of Michigan (1984); C.R. Wuest, PhD Thesis, University of California, Irvine (1983). 